\title{
Gigahertz Peaked Spectrum sources from the Jodrell Bank-VLA Astrometric Survey
}

\author{
I. Sources in the region $35^{\circ} \leq \delta \leq 75^{\circ}$ \\ A. Marecki $^{1}$, H. Falcke ${ }^{2}$, J. Niezgoda ${ }^{1}$, S.T. Garrington ${ }^{3}$, and A.R. Patnaik ${ }^{2}$ \\ 1 Centre for Astronomy, N. Copernicus University, ul. Gagarina 11, PL-87-100 Toruń, Poland \\ 2 Max-Planck-Institut für Radioastronomie, Auf dem Hügel 69, D-53121 Bonn, Germany \\ 3 Nuffield Radio Astronomy Laboratories, Jodrell Bank, Cheshire, SK11 9DL, UK
}

Received May 14; accepted October 13, 1998

\begin{abstract}
Observations with MERLIN ${ }^{1}$ at $408 \mathrm{MHz}$ have been used to establish the low-frequency part of the spectra of more than a hundred compact radio sources taken from the part of the Jodrell Bank-VLA Astrometric Survey limited by $35^{\circ} \leq \delta \leq 75^{\circ}$. These sources were selected from JVAS and other catalogues to have convex spectra between 1.4 and $8.4 \mathrm{GHz}$, characteristic of Gigahertz Peaked Spectrum (GPS) sources. We have confirmed convex shapes of the spectra of 76 objects (one half of our initial candidates) thereby yielding the largest genuine sample of GPS sources compiled so far. Seven of 17 identified quasars in the sample have large $(z \gtrsim 2)$ redshifts.
\end{abstract}

Key words: catalogs - galaxies: active - quasars: general - radio continuum: general

\section{Introduction}

By definition, Gigahertz Peaked Spectrum (GPS) sources have convex spectra with a turnover frequency $\nu_{\max }>$ $1 \mathrm{GHz}$. It is generally accepted that such a spectral shape results from synchrotron self-absorption due to a high compactness. Indeed, the linear sizes of GPS sources are very small $(10-1000 \mathrm{pc})$ and their radio luminosities are large $\left(L_{\text {radio }} \sim 10^{45} \mathrm{erg} / \mathrm{s}\right)$. An important feature which makes the GPS class particularly interesting, is that GPS objects identified with quasars often have very large redshifts. A review on the properties of GPS sources has been

Send offprint requests to: H. Falcke,

e-mail: hfalcke@mpifr-bonn.mpg.de

1 MERLIN is operated by The University of Manchester on behalf of the UK Particle Physics and Astronomy Research Council. given by O'Dea et al. (1991) — hereinafter OBS91 — and O'Dea (1998). De Vries et al. (1997) compiled spectra of 72 GPS sources and constructed a canonical GPS radio spectrum. They found it to have a constant shape independent of AGN type, redshift or radio luminosity.

As Gopal-Krishna \& Spoelstra (1993) pointed out, the great potential of GPS sources for discovering high$z$ objects continues to be the major motivation factor for enlarging the sample of GPS sources. A second motivation for increasing the number of known GPS sources comes from the discovery (Wilkinson et al. 1994) of a new class of Compact Symmetric Objects (CSO). Five archetypal CSOs (cf. Readhead et al. 1996a) are all acknowledged GPS sources. Readhead et al. (1996b) argue that CSOs are the young precursors of classical double radio sources. Increasing the list of known GPS sources offers a promising way to find more CSOs.

The search for GPS sources can be difficult because it requires both high resolution and high sensitivity observations at frequencies well below $1 \mathrm{GHz}$ and high sensitivity observations at $\nu>5 \mathrm{GHz}$, well above the potential peak of the spectrum. Therefore, unlike in the case of flat spectrum sources, only a modest number of GPS sources can simply be extracted from classical large catalogues e.g. the Green Bank (GB) surveys at $1.4 \mathrm{GHz}$ and $4.85 \mathrm{GHz}$ (White \& Becker 1992 and references therein - hereinafter WB92) and the Texas Survey at $365 \mathrm{MHz}$ (Douglas et al. 1996). For example, the sample of Stanghellini et al. (1990) was derived from the Texas Survey and the 1 Jy catalogue of Kühr et al. (1981a). Out of 55 sources and candidates they listed, 41 are currently acknowledged as GPS sources (O'Dea, priv. comm. 1996) and 33 make a complete sample (Stanghellini et al. 1996).

The lists published by Gopal-Krishna et al. (1983) and Spoelstra et al. (1985) resulting from dedicated observations, e.g. with the Westerbork Synthesis Radio Telescope 
(WSRT) or the Ooty telescope, are not very large: each one contains 25 sources (5 and 2 out of these two samples respectively had been retracted later). The sources gathered by the authors mentioned above have been collected in OBS91 in the so called "working sample" encompassing 95 objects. Gopal-Krishna \& Spoelstra (1993) confirmed the existence of 10 GPS sources and Cersosimo et al. (1994) found 7 more $^{2}$. On the other hand 6 sources from the list in OBS91, namely $0218+357,0528+134$, $0902+490,1851+488,2053-201$ and $2230+114$ proved to be "not so good" examples of the class and have been retracted. By the end of 1997 very few other GPS sources had been discovered.

There are two ways to achieve a "bulk" increase of this number. The first one has been followed by Snellen et al. (1998) - hereinafter SSB98 - the second is the basis of this paper. The former one is based on the Westerbork Northern Sky Survey (WENSS) being carried out with WSRT at 325 and $609 \mathrm{MHz}$. Naturally, the sources with inverted spectra in the WENSS are GPS candidates, which are followed up with observations at higher frequencies. This survey is particularly useful since it is the most sensitive survey at low frequencies (more than an order of magnitude better than the Texas Survey).

The published part of WENSS called "mini-survey" (Rengelink et al. 1997) is limited to $14^{\mathrm{h}} 10^{\mathrm{m}}<\alpha<$ $20^{\mathrm{h}} 30^{\mathrm{m}}, 57^{\circ}<\delta<72^{\circ} 50^{\prime}$ and covers $\sim 570$ square degrees only, so there are still large parts of the sky where this method cannot be applied. Nevertheless, SSB98 using the mini-survey plus an unpublished part of WENSS $\left(4^{\mathrm{h}}<\alpha<8^{\mathrm{h}} 30^{\mathrm{m}}\right.$, the same declination range as the minisurvey) were able to establish a list of 47 "new" GPS sources.

\section{Construction of the sample}

Since WENSS was not available when our programme started in 1994, we adopted a different approach which can be regarded as "opposite" to that of SSB98 because we started at the high frequencies end instead of the low one. Hence, to determine candidate GPS sources, we used $8.4 \mathrm{GHz}$ fluxes from the first part of the Jodrell Bank-VLA Astrometric Survey (JVAS) ${ }^{3}$ (Patnaik et al. 1992) containing all compact radio sources in the range $35^{\circ} \leq \delta \leq 75^{\circ}$ with fluxes $>200 \mathrm{mJy}$ at $5 \mathrm{GHz}$. We combined them with $1.4 \mathrm{GHz}$ and $4.85 \mathrm{GHz}$ fluxes from the GB catalogues (WB92). For 32 sources $1.4 \mathrm{GHz}$ fluxes were missing in WB92 so we substituted them with the catalogue flux density limit. Then we fitted the data with a second order polynomial of the form:

$\log S_{\nu}=S_{0}+\alpha \log \nu-c(\log \nu)^{2}$,

\footnotetext{
${ }^{2}$ Cersosimo et al. claim to discover more sources but only 7 have been finally recognised as GPS.

3 JVAS resulted from observations made with the VLA in "A" configuration.
}

Table 1. Subsample One

\begin{tabular}{|c|c|c|c|}
\hline $\begin{array}{l}\text { IAUname } \\
\text { (B1950) }\end{array}$ & \multicolumn{2}{|c|}{$\begin{array}{l}\text { R.A. } \\
\qquad(\mathrm{J} 2000)\end{array}$} & $\begin{array}{l}\text { Opt. } \\
\text { ID }\end{array}$ \\
\hline $0059+581$ & 010245.7630 & 582411.139 & $\mathrm{CW}$ \\
\hline $0102+480$ & 010549.9295 & 481903.183 & $\mathrm{EF}$ \\
\hline $0627+532$ & 063134.6860 & 531127.754 & BS \\
\hline $0652+426$ & 065610.6629 & 423702.751 & G \\
\hline $0652+577$ & 065712.5027 & 574156.740 & $\mathrm{EF}$ \\
\hline $0750+535$ & 075415.2177 & 532456.450 & $\mathrm{EF}$ \\
\hline $0828+493$ & 083223.2171 & 491321.036 & BL \\
\hline $1107+485$ & 111036.3237 & 481752.446 & $\mathrm{EF}$ \\
\hline $1239+552$ & 124127.7043 & 545819.040 & $\mathrm{EF}$ \\
\hline $1256+546$ & 125815.6078 & 542152.112 & $\mathrm{EF}$ \\
\hline $1311+552$ & 131337.8518 & 545823.894 & $\mathrm{EF}$ \\
\hline $1428+422$ & 143023.7418 & 420436.503 & $\mathrm{EF}$ \\
\hline $1532+680$ & 153243.3426 & 675513.992 & $\mathrm{EF}$ \\
\hline $1602+576$ & 160355.9311 & 573054.415 & $\mathrm{BS}$ \\
\hline $1627+476$ & 162837.5064 & 473410.414 & $\mathrm{BS}$ \\
\hline $1630+358$ & 163231.2578 & 354737.740 & $\mathrm{EF}$ \\
\hline $1745+670$ & 174554.3577 & 670349.302 & $\mathrm{EF}$ \\
\hline $1755+578$ & 175603.6285 & 574847.990 & $\mathrm{BS}$ \\
\hline $1815+614$ & 181536.7920 & 612711.641 & $\mathrm{EF}$ \\
\hline $1946+708$ & 194553.5197 & 705548.723 & $?$ \\
\hline $2253+417$ & 225536.7082 & 420252.535 & $\mathrm{BS}$ \\
\hline $2310+385$ & 231258.7950 & 384742.668 & $\mathrm{BS}$ \\
\hline $2323+478$ & 232544.9131 & 480625.280 & $\mathrm{BS}$ \\
\hline $2356+385$ & 235933.1809 & 385042.322 & $\mathrm{BS}$ \\
\hline
\end{tabular}

Explanation of ID field:

BL: BL Lac object

BS: blue stell. obj. (without the spec. - basically a quasar) BG: blue galaxy?

CW: crowded field - too many objects for a clean identification

EF: empty field

G: galaxy

NS: neutral stell. obj. (same magn. in both $\mathrm{E}$ and $\mathrm{O}$ prints) OB: obscured field

Q: quasar

RS: red stellar object.

where $\nu$ is the observed frequency in $\mathrm{GHz}$ and $S_{\nu}$ the flux density in mJy and selected the sources with convex spectral shapes. The criterion for selecting an initial list of candidate GPS sources was that the curvature $c$ had to be greater than 1 with a peak in the spectrum in the observable range (the limit of $c>1$ is rather conservative and includes also spectra which are much flatter than those of typical GPS sources). Admittedly, although this criterion seems to be conservative enough, a few sources from the OBS91 "working sample" which happen to be JVAS members, and therefore have been fitted with polynomials $(0018+729,0248+430,0552+398,2015+657,2021+614$, $2352+495)$, did not fulfill it. In the end, the $c>1$ threshold was chosen as a good compromise, to produce a reasonably sized sample for future observations. 
The number of the initial candidates selected with the above procedure was 163 .

This list contained 14 known GPS sources: $0108+388$, $0153+744,0636+680,0646+600,0710+439,0711+356$, $1031+567,1225+368,1333+459,1843+356$ and $2050+364$ listed in OBS91; 0903+684 from Gopal-Krishna \& Spoelstra (1993) and two discovered by Snellen et al. (1995) - $0700+470$ and $1324+574$. These were not considered for further observations.

We divided the remaining sample of 149 sources into two parts. The first one - hereinafter "Subsample One" - contains those which are found in the $365 \mathrm{MHz}$ Texas survey (24 sources). They are listed in Table 1 along with JVAS positions rounded to 1 milliarcsecond and our identifications made using POSS. Nine of these are also listed in the $6 \mathrm{C}$ catalogue which has a flux density limit of $200 \mathrm{mJy}$ at $151 \mathrm{MHz}$ (Hales et al. 1993 and references therein).

For the remaining 125 sources - hereinafter "Subsample Two" — we had virtually no low frequency data. Although 19 of these sources were present in the B3 survey at $408 \mathrm{MHz}$ (Ficarra et al. 1985), its overlap in sky coverage with the first part of the JVAS survey we use here is small and, furthermore, the B3 survey has significant errors in flux density near the catalogue limit of $100 \mathrm{mJy}$.

The aforementioned crude extrapolation of the spectra applied to medium and high frequency data $(1.4 \mathrm{GHz}$ $<\nu<8.4 \mathrm{GHz}$ ) of Subsample Two plus the flux density limit of the Texas Survey gave us only an unreliable estimate of the frequency turnover and even the convex shape of the spectrum remained uncertain in some cases. Particularly some weaker sources could either be GPS or mere flat spectrum depending on whether their $365 \mathrm{MHz}$ fluxes were far below or just below the Texas catalogue limit respectively. Another effect that will produce a spurious convex shape in our non-simultaneous data is the variability typically observed in flat spectrum sources.

\section{Observations and data reduction}

In order to investigate the low frequency part of the spectra of Subsample Two sources we set up a programme of flux density measurements at low frequency with a high resolution facility. We used MERLIN at $408 \mathrm{MHz}$ because of its superior resolving capability - $1^{\prime \prime}$. The observations were carried out in the period from November 1994 until January 1995 and each source was typically observed twice (with different hour angles) for about 15 minutes per scan.

Such short snapshots cannot be used to produce reliable maps with MERLIN, since the aperture coverage is too sparse. Confusion is a significant effect at this low frequency and therefore fringe-frequency vs. delay (FFD) plots were used to separate confusing sources from the central target source (see e.g. Walker 1981). Since the data were not phase-calibrated the coherence time is limited to a few minutes. By taking $128 \times 4$ second sub-samples of the data and Fourier transforming them in both time and frequency, a "map" of the field surrounding the source can be produced whose axes are fringe-frequency and delay. The target source will have close to zero fringe-frequency and delay, since its position is well known from the JVAS survey, and appear at the centre of the "map", while confusing sources will be offset from the centre. The flux density of the target source was determined from the central peak in the FFD map. The FFD plots were inspected visually; the detection threshold in a single plot was set at $3 \sigma$ and flux density values were only listed if the target was detected in two or more plots. In all cases, the values determined from the FFD plots were consistent with simple averages of phase-calibrated data on individual baselines calculated using the Astronomical Image Processing System (AIPS). The flux density scale was determined using observations of $3 \mathrm{C} 286$, for which the Baars et al. (1977) value was used.

In Table 2 we report the results of successful measurements of $408 \mathrm{MHz}$ fluxes for 98 sources from Subsample Two along with their JVAS positions rounded to 1 milliarcsecond and our identifications made using POSS. Interference and other problems at other sites resulted in the loss of data for the remaining 27 sources. They are listed in Table 3 . The flux density values given in Table 2 resulted from averaging the measurements over all baselines involving the Defford or Knockin telescopes (6 or 8 baselines, depending on whether the Lovell telescope was used or not) and in both LL and RR polarisations. Shorter baselines were not used because they provide insufficient resolution in delay or fringe-frequency to separate the confusing sources; additionally the use of longer baselines reduces any contribution from large-scale halos seen around some GPS sources. The longest baselines involving the Cambridge telescope were not used because interference limited the useful bandwidth to $1.5 \mathrm{MHz}$, rather than the $4 \mathrm{MHz}$ used elsewhere. Because baseline combinations and the volume of data per source varied form source to source the errors range form 10 to $30 \mathrm{mJy}$.

\section{The new sample of GPS spectrum sources}

Finally we merged Subsample One (24 sources) with those 98 sources from Subsample Two whose flux densities we had successfully measured with MERLIN at $408 \mathrm{MHz}$. Since the selection process for this project was carried out, the $5 \mathrm{GHz}$ Green Bank survey of WB92 has been superseded by the GB6 survey (Gregory et al. 1996) and at 1.4 GHz we can now use the the NRAO VLA Sky Survey $(\text { NVSS })^{4}$ (Condon et al. 1998) which has a resolution of $45^{\prime \prime}$ and an rms sensitivity of approximately $1.5 \mathrm{mJy}$.

\footnotetext{
${ }^{4}$ available at ftp://nvss.cv.nrao.edu/pub/nvss/CATALOG
} 
Table 2. Subsample Two - flux densities of sources at $408 \mathrm{MHz}$

\begin{tabular}{|c|c|c|c|c|}
\hline $\begin{array}{l}\text { IAU name } \\
\text { (B1950) }\end{array}$ & \multicolumn{2}{|c|}{$(\mathrm{J} 2000)$} & $\begin{array}{c}\text { Flux } \\
{[\mathrm{mJy}]}\end{array}$ & $\begin{array}{r}\text { Opt. } \\
\text { ID }\end{array}$ \\
\hline $0001+478$ & 000346.0413 & 480704.134 & 104 & BS \\
\hline $0015+529$ & 001751.7596 & 531219.126 & 100 & $\mathrm{BS}$ \\
\hline $0046+511$ & 004937.9901 & 512813.700 & 123 & NS \\
\hline $0051+679$ & 005417.6237 & 681111.175 & 127 & OB \\
\hline $0051+706$ & 005417.6884 & 705356.625 & 260 & NS \\
\hline $0058+498$ & $\begin{array}{llll}01 & 01 & 16.9988\end{array}$ & 500444.991 & 125 & $\mathrm{BS}$ \\
\hline $0102+511$ & 010529.5588 & 512546.576 & 262 & NS \\
\hline $0123+731$ & 012704.7169 & 732312.676 & 330 & $\mathrm{EF}$ \\
\hline $0129+431$ & 013244.1273 & 432532.667 & 244 & BS \\
\hline $0129+560$ & 013220.4503 & 562040.372 & 113 & $\mathrm{CW}$ \\
\hline $0140+490$ & 014346.8791 & 491541.586 & 105 & $\mathrm{CW}$ \\
\hline $0148+546$ & 015136.2876 & 545437.688 & 100 & $\mathrm{EF}$ \\
\hline $0153+389$ & 015631.4088 & 391430.929 & 119 & BS \\
\hline $0213+444$ & $0216 \quad 17.1707$ & 443743.405 & 154 & $\mathrm{EF}$ \\
\hline $0251+393$ & 025442.6316 & 393134.714 & 114 & $\mathrm{BS}$ \\
\hline $0307+380$ & 031049.8805 & $38 \quad 1453.845$ & 148 & NS \\
\hline $0335+599$ & 033909.3942 & 600856.960 & 134 & $\mathrm{RS}$ \\
\hline $0336+473$ & $0340 \quad 10.7897$ & 473227.328 & 103 & BS \\
\hline $0338+480$ & $03 \quad 42 \quad 10.3522$ & 480946.948 & 113 & NS \\
\hline $0412+447$ & 041556.5246 & 445249.676 & 110 & OB \\
\hline $0424+414$ & 042746.0455 & 413301.091 & 272 & $\mathrm{EF}$ \\
\hline $0454+550$ & 045854.8417 & 550842.042 & 192 & BS \\
\hline $0513+714$ & 051928.8835 & 713303.740 & 153 & $\mathrm{EF}$ \\
\hline $0514+474$ & $05 \quad 1812.0899$ & 473055.536 & 220 & $\mathrm{CW}$ \\
\hline $0533+446$ & 053730.0630 & 444103.533 & 155 & $?$ \\
\hline $0559+422$ & 060258.9438 & $42 \quad 1209.999$ & 259 & $\mathrm{BS}$ \\
\hline $0610+510$ & $06 \quad 1449.1589$ & $\begin{array}{lll}51 & 02 & 13.124\end{array}$ & 143 & $\mathrm{BG}$ \\
\hline $0621+446$ & 062518.2652 & 444001.628 & 101 & BS \\
\hline $0630+497$ & 063352.2068 & 494345.939 & 149 & BS \\
\hline $0651+410$ & 065510.0243 & 410010.148 & 130 & G \\
\hline $0655+696$ & 070106.6159 & 693629.414 & 293 & $\mathrm{BS}$ \\
\hline $0708+742$ & 071436.1236 & $7408 \quad 10.142$ & 105 & $\mathrm{BS}$ \\
\hline $0713+669$ & 071805.6314 & 665153.332 & 145 & $\mathrm{EF}$ \\
\hline $0718+374$ & 072201.2600 & 372228.628 & 64 & BS \\
\hline $0732+755$ & 073913.1962 & $\begin{array}{lll}75 & 27 & 47.702\end{array}$ & 141 & $\mathrm{EF}$ \\
\hline $0753+373$ & 075628.2513 & 371455.647 & 59 & $\mathrm{BS}$ \\
\hline $0753+519$ & 075659.5457 & 515100.237 & 98 & $\mathrm{BS}$ \\
\hline $0758+594$ & 080224.5932 & 592134.800 & 102 & $\mathrm{BS}$ \\
\hline $0849+675$ & 085334.3220 & 672215.665 & 212 & BS \\
\hline $0851+719$ & 085654.8695 & 714623.894 & 105 & BS \\
\hline $0900+520$ & 090358.5758 & 515100.658 & 354 & $?$ \\
\hline $0924+732$ & 092942.1565 & 730404.553 & 114 & G \\
\hline $0925+745$ & 093053.7823 & 742005.930 & 112 & $\mathrm{EF}$ \\
\hline $0939+620$ & 094314.5025 & 615033.343 & 99 & $\mathrm{BS}$ \\
\hline $1017+436$ & 102027.2021 & 432056.342 & 51 & BS \\
\hline $1019+429$ & 102213.1324 & 423925.618 & 138 & $\mathrm{RS}$ \\
\hline $1032+509$ & 103506.0176 & 504006.087 & 198 & $\mathrm{EF}$ \\
\hline $1035+430$ & 103818.1899 & 424442.766 & 194 & $\mathrm{RS}$ \\
\hline $1043+541$ & 104624.0372 & 535426.220 & 181 & $\mathrm{BS}$ \\
\hline $1055+433$ & 105802.9208 & 430441.505 & 182 & $\mathrm{EF}$ \\
\hline $1101+609$ & 110453.6946 & 603855.287 & 151 & $\mathrm{BS}$ \\
\hline $1125+366$ & 112758.8707 & 362028.352 & 86 & $\mathrm{BS}$ \\
\hline $1138+644$ & 114112.2283 & 641005.484 & 180 & $\mathrm{EF}$ \\
\hline
\end{tabular}

Table 2. continued

\begin{tabular}{|c|c|c|c|c|}
\hline $\begin{array}{l}\text { IAU name } \\
(\mathrm{B} 1950)\end{array}$ & \multicolumn{2}{|c|}{ (J2000) } & $\begin{array}{c}\text { Flux } \\
{[\mathrm{mJy}]}\end{array}$ & $\begin{array}{r}\text { Opt. } \\
\text { ID }\end{array}$ \\
\hline $1157+532$ & 120006.0107 & 530037.118 & 188 & $\mathrm{Q}$ \\
\hline $1206+415$ & 120922.7884 & 411941.369 & 152 & BS \\
\hline $1226+638$ & 122906.0256 & 633500.986 & 153 & $\mathrm{RS}$ \\
\hline $1232+366$ & 123505.8076 & $\begin{array}{lll}36 & 21 & 19.308\end{array}$ & 121 & $\mathrm{BS}$ \\
\hline $1239+606$ & 124129.5907 & 602041.320 & 136 & $\mathrm{BS}$ \\
\hline $1245+676$ & 124733.3300 & 672316.457 & 107 & $\mathrm{G}$ \\
\hline $1300+485$ & 130217.1974 & 481917.572 & 163 & $\mathrm{BS}$ \\
\hline $1308+471$ & 131053.5906 & 465352.219 & 160 & $\mathrm{EF}$ \\
\hline $1320+394$ & 132255.6615 & $\begin{array}{lll}39 & 12 & 07.984\end{array}$ & 119 & $\mathrm{BS}$ \\
\hline $1321+410$ & 132412.0940 & $4048 \quad 11.773$ & 92 & $\mathrm{BS}$ \\
\hline $1337+637$ & 133923.7812 & 632858.425 & 185 & BS \\
\hline $1338+381$ & 134022.9519 & 375443.839 & 105 & $\mathrm{RS}$ \\
\hline $1357+404$ & 135938.0943 & 401138.260 & 129 & $\mathrm{EF}$ \\
\hline $1403+411$ & 140507.7949 & 405657.847 & 134 & $\mathrm{BS}$ \\
\hline $1454+447$ & 145554.1361 & 443137.668 & 115 & $\mathrm{BS}$ \\
\hline $1533+487$ & 153514.6540 & 483659.697 & 122 & $\mathrm{BS}$ \\
\hline $1534+501$ & 153552.0395 & 495739.084 & 103 & $\mathrm{BS}$ \\
\hline $1544+398$ & 154553.2331 & 394146.857 & 168 & G \\
\hline $1607+563$ & 160820.7518 & 561356.373 & 129 & BS \\
\hline $1614+466$ & 161603.7667 & 463225.231 & 98 & $\mathrm{BS}$ \\
\hline $1722+611$ & 172240.0578 & 610559.801 & 213 & $\mathrm{BS}$ \\
\hline $1724+609$ & 172441.4142 & 605555.731 & 179 & $\mathrm{EF}$ \\
\hline $1753+648$ & 175407.5904 & 645202.642 & 89 & $\mathrm{BS}$ \\
\hline $1801+459$ & $1802 \quad 25.1427$ & 455734.645 & 192 & $\mathrm{BS}$ \\
\hline $1812+560$ & 181257.6692 & 560349.198 & 193 & $\mathrm{BS}$ \\
\hline $1820+397$ & 182159.6991 & 394559.647 & 743 & $\mathrm{BS}$ \\
\hline $1822+682$ & 182159.4951 & 681843.003 & 192 & $\mathrm{BS}$ \\
\hline $1828+399$ & 182956.5203 & 395734.690 & 116 & $\mathrm{EF}$ \\
\hline $1839+389$ & 184057.1550 & 390045.712 & 133 & $\mathrm{BS}$ \\
\hline $1839+548$ & 184057.3780 & 545215.920 & 121 & $\mathrm{BS}$ \\
\hline $1937+630$ & 193816.1680 & $\begin{array}{llll}6307 & 17.803\end{array}$ & 254 & $?$ \\
\hline $1939+429$ & 194049.3198 & 430424.671 & 197 & $\mathrm{BS}$ \\
\hline $1941+413$ & 194258.6385 & 412923.073 & 167 & $\mathrm{BS}$ \\
\hline $2000+472$ & 200210.4183 & 472528.777 & 150 & CW \\
\hline $2005+642$ & 200617.6949 & 642445.423 & 137 & $\mathrm{RS}$ \\
\hline $2013+508$ & 201428.5899 & 505909.532 & 174 & $\mathrm{EF}$ \\
\hline $2014+463$ & 201539.9865 & 462850.886 & 122 & CW \\
\hline $2112+374$ & 211444.1230 & 374225.719 & 181 & CW \\
\hline $2119+709$ & 211954.1676 & 711036.091 & 147 & $\mathrm{EF}$ \\
\hline $2151+431$ & 215350.9585 & 432254.497 & 110 & NS \\
\hline $2202+716$ & 220330.4694 & 715108.527 & 200 & $\mathrm{RS}$ \\
\hline $2248+555$ & 225042.8496 & 555014.608 & 212 & $\mathrm{G}$ \\
\hline $2300+638$ & 230241.3165 & 640552.858 & 117 & $\mathrm{EF}$ \\
\hline $2310+724$ & 231219.6998 & 724126.924 & 231 & $\mathrm{EF}$ \\
\hline $2341+697$ & 234343.7360 & $\begin{array}{llll}70 & 03 & 19.398\end{array}$ & 160 & NS \\
\hline
\end{tabular}

Explanation of ID field is given in Table 1. 
Table 3. Subsample Two - sources not measured at $408 \mathrm{MHz}$

\begin{tabular}{|c|c|c|c|}
\hline $\begin{array}{l}\text { IAU name } \\
\text { (B1950) }\end{array}$ & \multicolumn{2}{|c|}{$\begin{array}{l}\text { R.A. } \\
\qquad(\mathrm{J} 2000)\end{array}$} & $\begin{array}{l}\text { Opt. } \\
\text { ID }\end{array}$ \\
\hline $0310+435$ & 031408.0539 & 434519.770 & $\mathrm{EF}$ \\
\hline $0314+696$ & 031922.0734 & 694925.603 & $\mathrm{EF}$ \\
\hline $0418+437$ & 042152.0619 & 435304.216 & CW \\
\hline $0537+392$ & $05 \quad 4044.4377$ & 391612.236 & $\mathrm{RS}$ \\
\hline $0601+578$ & 060542.2275 & 575316.351 & $\mathrm{BS}$ \\
\hline $0638+528$ & 064227.8215 & 524759.282 & $\mathrm{BS}$ \\
\hline $0644+491$ & 064847.1190 & 490720.736 & $\mathrm{BS}$ \\
\hline $0651+428$ & 065443.5263 & 424758.728 & G \\
\hline $0903+669$ & 090723.5240 & 664446.942 & $\mathrm{EF}$ \\
\hline $1238+702$ & 124034.6989 & 695830.616 & $\mathrm{BS}$ \\
\hline $1245+716$ & 124709.3270 & 712420.018 & $\mathrm{EF}$ \\
\hline $1341+691$ & 134300.5520 & 685517.160 & BS \\
\hline $1406+564$ & 140812.9466 & $\begin{array}{lll}56 & 13 & 32.488\end{array}$ & BS \\
\hline $1436+445$ & 143828.5048 & 441812.085 & $\mathrm{BS}$ \\
\hline $1447+536$ & 144859.1739 & 532609.282 & $\mathrm{EF}$ \\
\hline $1456+375$ & 145844.7949 & 372021.627 & $\mathrm{BS}$ \\
\hline $1526+670$ & 152642.8732 & 665054.617 & $\mathrm{NS}$ \\
\hline $1550+582$ & 155158.2077 & 580644.466 & $\mathrm{BS}$ \\
\hline $1611+425$ & 161304.8038 & 422318.903 & $\mathrm{BS}$ \\
\hline $1622+665$ & 162304.5221 & 662401.084 & G \\
\hline $1924+420$ & 192631.0504 & 420958.991 & G \\
\hline $2119+664$ & 212046.2045 & 664220.216 & $\mathrm{EF}$ \\
\hline $2132+406$ & 213424.1053 & 405011.345 & $\mathrm{EF}$ \\
\hline $2230+625$ & 223222.8655 & 624936.436 & $\mathrm{OB}$ \\
\hline $2236+678$ & 223815.0284 & 680459.758 & $\mathrm{OB}$ \\
\hline $2249+402$ & 225159.7715 & 403058.155 & $\mathrm{BS}$ \\
\hline $2351+550$ & 235342.3011 & $\begin{array}{lll}55 & 18 & 40.670\end{array}$ & $\mathrm{BS}$ \\
\hline
\end{tabular}

Explanation of ID field is given in Table 1.

Unfortunately, at the time of writing, at the time of writin, NVSS - although almost complete — did not cover all areas of the sky within its declination limits. Many NVSS maps $\left(4^{\circ} \times 4^{\circ}\right.$ each $)$ appear to be "patchy" and the "holes" can sometimes be quite large. Our survey suffered considerably from this shortcoming of the current edition of NVSS - 9 sources out of those 122 sources we wanted to study were simply not present in the NVSS catalogue. (One source out of these nine was also unavailable in GB6.) Additionally we decided to remove 2 other sources from the further processing: one of these is blended with a nearby source and the second one has an extended structure which should be studied in more detail.

At $1.4 \mathrm{GHz}$ we also tried to use the Faint Images of Radio Sky at Twenty (FIRST) catalogue ${ }^{5}$ (White et al. 1997) - 24 our sources could be found there. For 20 objects out of these we noted a very good compatibility between FIRST and NVSS based fluxes; the 4 objects which showed discrepancy are indicated in Table 6 .

The selection process described above gave us finally 111 objects for which we arrayed the flux density values at

\footnotetext{
5 available at http://sundog.stsci.edu/
}

each frequency. Then we attempted to fit model spectra to the available data using a broken power-law with the following formula (Moffet 1975):

$$
S(\nu)=\frac{S_{0}}{1-\mathrm{e}^{-1}} \cdot\left(\nu / \nu_{0}\right)^{k} \cdot\left(1-\mathrm{e}^{-\left(\nu / \nu_{0}\right)^{l-k}}\right) .
$$

Here $k$ and $l$ are the spectral indices of the rising and declining parts of the spectrum as often used in radio astronomy, while $S_{0}$ and $\nu_{0}$ are just fitting parameters which are not equal to the maximum flux density $\left(S_{\max }\right)$ and the peak frequency $\left(\nu_{\max }\right)$ of the fitted spectrum. Even though the broken power-law seems to be the physically more sensible choice for a model spectrum compared to a simple second-order polynomial, it has the disadvantage that it is unconstrained if the peak of the spectrum falls beyond the 2nd highest or below the 2nd lowest available frequency. In these cases we fixed the peak of the model spectrum (i.e. $S_{\max }$ and $\nu_{\max }$ ) at the peak of the measured data - this was usually the measurement at $4.85 \mathrm{GHz}$ - and marked the fit as unconstrained in Table 4. This means that the values for $k$ or $l$ have to be considered as a lower or upper limit respectively (i.e. in reality the spectrum will be more inverted at low frequencies or steeper at high frequencies).

The spectra of 35 sources could not be fitted with such a convex-shaped curve (Fig. 1) and we claim that those sources cannot be termed "GPS sources" at all and most likely are just variable flat-spectrum sources. The 76 spectra that could be fitted with our algorithm are presented in Fig. 2 and the fitting parameters are given in Table 4. As can be seen from Fig. 2 and Table 4, some of the sources with unconstrained model spectra, fit the data relatively poorly at low frequencies or have relatively flat spectral indices (e.g. $0307+380$ and $0610+510$ ) and thus are less probable GPS candidates.

In Table 5 we specified some parameters of our "new" GPS sources gathered from the literature: the names of other catalogues a particular source is a member, the optical identification according to the NASA/IPAC Extragalactic Database (NED) and the redshift. At the time of writing 21 objects from our collection have been identified (3 galaxies ${ }^{6}, 18$ QSOs) and their redshifts are known. Those 3 galaxies have low redshifts $(z \lesssim 0.1)$; on the other hand - as expected - the majority of quasars have large redshifts: for 6 QSOs $1<z \lesssim 2$, for 7 other QSOs $z \gtrsim 2$. One QSO, 1338+381, is extremely redshifted: $z=3.103$.

Most of the GPS sources studied so far hardly show any variability, therefore we checked our sources against possible flux variations. Firstly, because a significant variability of the flux density would mean that the source in question is likely not to be a GPS and secondly -

\footnotetext{
${ }^{6}$ It is worth noting that our identifications shown in Tables 1 and 2 yielded 4 galaxies. The identifications for $0651+410$ and $1245+676$ are given by NED, for the two other ones - $1544+398$ and $2248+555$ - are not.
} 
Table 4. GPS sources' spectra fitting parameters

\begin{tabular}{|c|c|c|c|c|c|c|c|c|}
\hline Number & B1950name & $S_{0}$ & $\nu_{0}$ & $k$ & $l$ & $S_{\max }$ & $\nu_{\max }$ & unconstrained \\
\hline 1 & $0001+478$ & 286. & 3.66 & +0.671 & -2.1 & 332. & 2.68 & \\
\hline 2 & $0015+529$ & 657. & 3.27 & +1.13 & -0.381 & 687. & 4.82 & \\
\hline 3 & $0046+511$ & 246. & 7.14 & +0.549 & -1.63 & 284. & 4.85 & $\bullet$ \\
\hline 4 & $0051+679$ & 324. & 6.97 & +0.577 & -1.85 & 379. & 4.85 & $\bullet$ \\
\hline 5 & $0058+498$ & 187. & 5.94 & +0.322 & -1.76 & 237. & 3.49 & \\
\hline 6 & $0102+480$ & 1110. & 3.03 & +1.06 & -0.774 & 1110. & 3.01 & \\
\hline 7 & $0102+511$ & 530. & 1.85 & +0.775 & -1.33 & 564. & 1.42 & $\bullet$ \\
\hline 8 & $0123+731$ & 361. & 3.25 & +0.264 & -1.27 & 449. & 1.63 & \\
\hline 9 & $0129+560$ & 659. & 3.42 & +1.05 & -0.956 & 662. & 3.14 & \\
\hline 10 & $0140+490$ & 228. & 4.28 & +0.524 & -1.53 & 262. & 2.85 & \\
\hline 11 & $0148+546$ & 137. & 8.39 & +0.256 & -1.86 & 181. & 4.73 & \\
\hline 12 & $0153+389$ & 203. & 7.44 & +0.404 & -2.17 & 257. & 4.85 & $\bullet$ \\
\hline 13 & $0307+380$ & 581. & 6.21 & +0.862 & -1.66 & 628. & 4.85 & $\bullet$ \\
\hline 14 & $0335+599$ & 192. & 7.92 & +0.309 & -2.12 & 251. & 4.85 & $\bullet$ \\
\hline 15 & $0336+473$ & 295. & 2.96 & +0.762 & -0.886 & 301. & 2.43 & \\
\hline 16 & $0412+447$ & 277 . & 7.84 & +0.349 & +0.349 & & & \\
\hline 17 & $0454+550$ & 257. & 7.85 & +0.302 & -2.23 & 340. & 4.85 & $\bullet$ \\
\hline 18 & $0513+714$ & 219. & 3.26 & +0.394 & -1.19 & 253. & 1.91 & \\
\hline 19 & $0514+474$ & 863. & 2.05 & +1.13 & -0.842 & 863. & 2.02 & \\
\hline 20 & $0610+510$ & 143. & 9.9 & +0.157 & -1.74 & 198. & 4.85 & $\bullet$ \\
\hline 21 & $0627+532$ & 832. & 0.767 & +0.996 & -0.593 & 835. & 0.836 & \\
\hline 22 & $0630+497$ & 183. & 7.51 & +0.245 & -2.89 & 255. & 4.85 & $\bullet$ \\
\hline 23 & $0651+410$ & 408. & 6.57 & +0.661 & -1.06 & 431. & 4.85 & • \\
\hline 24 & $0652+577$ & 528. & 0.564 & +1.01 & -0.649 & 528. & 0.592 & \\
\hline 25 & $0655+696$ & 481. & 1.63 & +0.69 & -0.644 & 484. & 1.42 & $\bullet$ \\
\hline 26 & $0718+374$ & 277. & 6.62 & +0.692 & -1.43 & 302. & 4.85 & $\bullet$ \\
\hline 27 & $0750+535$ & 602. & 2.26 & +0.57 & -1.44 & 678. & 1.55 & \\
\hline 28 & $0753+373$ & 287. & 2.85 & +1.05 & -1.15 & 292. & 2.5 & \\
\hline 29 & $0753+519$ & 221. & 3.66 & +0.58 & -1.22 & 242. & 2.52 & \\
\hline 30 & $0758+594$ & 221. & 2.84 & +0.635 & -0.711 & 225. & 2.27 & \\
\hline 31 & $0849+675$ & 308. & 2.72 & +0.44 & -1.11 & 347. & 1.66 & \\
\hline 32 & $0925+745$ & 284. & 4.95 & -1.07 & +0.554 & 287. & 4.26 & \\
\hline 33 & $1017+436$ & 212. & 6.49 & +0.728 & -2.22 & 246. & 4.85 & • \\
\hline 34 & $1019+429$ & 277. & 7.5 & +0.496 & -1.3 & 314. & 4.85 & • \\
\hline 35 & $1032+509$ & 180. & 7.71 & +0.124 & -1.16 & 245. & 2.81 & \\
\hline 36 & $1055+433$ & 267. & 0.69 & +1.44 & -0.237 & 308. & 1.42 & $\bullet$ \\
\hline 37 & $1107+485$ & 712. & 0.69 & +0.645 & -0.845 & 734. & 0.528 & \\
\hline 38 & $1125+366$ & 70.4 & 0.347 & +0.292 & +2.14 & & & \\
\hline 39 & $1138+644$ & 228. & 9.59 & +0.192 & -1.68 & 307. & 4.85 & $\bullet$ \\
\hline 40 & $1206+415$ & 449. & 10.4 & -2.04 & +0.475 & 492. & 7.29 & \\
\hline 41 & $1226+638$ & 422. & 2.89 & +0.751 & -1.24 & 446. & 2.21 & \\
\hline 42 & $1232+366$ & 198. & 8.41 & +0.372 & -1.29 & 234. & 4.85 & $\bullet$ \\
\hline 43 & $1239+606$ & 435. & 1.41 & +1.31 & -0.93 & 435. & 1.42 & $\bullet$ \\
\hline 44 & $1245+676$ & 258. & 1.16 & +1.29 & -0.561 & 264. & 1.42 & $\bullet$ \\
\hline 45 & $1256+546$ & 1090. & 0.539 & +0.901 & -0.925 & 1110. & 0.471 & \\
\hline 46 & $1311+552$ & 1980. & 0.555 & +1.33 & -0.851 & 1980. & 0.578 & \\
\hline 47 & $1321+410$ & 476. & 2.83 & +1.09 & -0.98 & 478. & 2.62 & \\
\hline 48 & $1338+381$ & 330. & 3.27 & +0.771 & -1.21 & 347. & 2.53 & \\
\hline 49 & $1357+404$ & 356. & 12.1 & +0.349 & +0.349 & & & \\
\hline 50 & $1403+411$ & 223. & 6.71 & +0.346 & -1.58 & 276. & 3.92 & \\
\hline
\end{tabular}


Table 4. continued

\begin{tabular}{rlrlccccc}
\hline Number & B1950name & $S_{0}$ & $\nu_{0}$ & $k$ & $l$ & $S_{\max }$ & $\nu_{\max }$ & unconstrained \\
\hline 51 & $1454+447$ & 206. & 2.36 & +0.588 & -0.407 & 206. & 2.43 & \\
52 & $1532+680$ & 455. & 2.57 & +0.329 & -1.32 & 551. & 1.42 & $\bullet$ \\
53 & $1534+501$ & 341. & 6.97 & +0.584 & -1.09 & 367. & 4.85 & $\bullet$ \\
54 & $1544+398$ & 221. & 6.4 & +0.363 & -4.67 & 310. & 4.85 & $\bullet$ \\
55 & $1607+563$ & 261. & 3.54 & +0.538 & -0.797 & 273. & 2.48 & \\
56 & $1627+476$ & 219. & 3.06 & +0.241 & -1.11 & 271. & 1.42 & $\bullet$ \\
57 & $1630+358$ & 466. & 2.5 & +0.377 & -1.11 & 537. & 1.42 & $\bullet$ \\
58 & $1745+670$ & 548. & 2.7 & +0.266 & -1.46 & 696. & 1.42 & $\bullet$ \\
59 & $1753+648$ & 220. & 7.26 & +0.519 & -1.66 & 257. & 4.85 & $\bullet$ \\
60 & $1755+578$ & 785. & 2.07 & +1.06 & -1.07 & 793. & 1.86 & \\
61 & $1801+459$ & 231. & 2.78 & +0.337 & -0.77 & 256. & 1.46 & \\
62 & $1815+614$ & 580. & 3.9 & +0.0731 & -1.69 & 852. & 1.61 & \\
63 & $1820+397$ & 753. & 0.457 & -0.519 & +0.527 & 761. & 0.575 & \\
64 & $1839+548$ & 240. & 5.62 & +0.436 & -0.983 & 265. & 3.42 & \\
65 & $1946+708$ & 965. & 1.92 & +0.818 & -0.755 & 970. & 1.72 & \\
66 & $2000+472$ & 946. & 4.11 & +0.996 & -0.588 & 949. & 4.51 & \\
67 & $2005+642$ & 227. & 0.502 & +0.346 & +3.4 & & & \\
68 & $2013+508$ & 363. & 1.67 & +0.849 & -0.927 & 369. & 1.42 & \\
69 & $2014+463$ & 153. & 8.03 & +0.231 & -1.88 & 205. & 4.44 & \\
70 & $2119+709$ & 167. & 9.07 & +0.217 & -1.79 & 224. & 4.85 & $\bullet$ \\
71 & $2151+431$ & 257. & 3.25 & +0.63 & -0.917 & 268. & 2.41 & \\
72 & $2248+555$ & 469. & 4.72 & +0.512 & -0.633 & 481. & 3.43 & \\
73 & $2253+417$ & 1840. & 1.83 & +0.706 & -0.909 & 1900. & 1.44 & \\
74 & $2310+385$ & 682. & 3.2 & +0.6 & -1.14 & 735. & 2.25 & \\
75 & $2341+697$ & 155. & 7.5 & +0.147 & -1.59 & 213. & 3.45 & \\
76 & $2356+385$ & 560. & 3.85 & +0.524 & -1.34 & 632. & 2.55 & \\
\hline & & & & & & & & \\
\end{tabular}

since our data are not simultaneous — any variability makes derivation of spectra questionable. The part of sources' spectra around $1.4 \mathrm{GHz}$ is obviously the most "sensitive" with regard to the GPS phenomenon so we compared fluxes at this frequency given in WB92 to those from NVSS. We applied corrections for the different beam sizes of these two measurements. If a particular source had changed its flux between epochs of the GB surveys and NVSS/FIRST more than $25 \%$ or the $1.4 \mathrm{GHz}$ GB flux was missing in WB92 we treated such a source as potentially variable, unless we could find a second epoch flux density measurement elsewhere. We assigned a "candidate" status for such objects and listed them in Table 6. Among these there are 4 sources $(0412+447,1125+366,1357+404,2005+642)$ with inverted spectra only, i.e. apparently having turnovers in their spectra at frequencies larger than 8.4 GHz. This feature was yet another reason to assign them a candidate status.

\section{Notes on individual sources}

Apart from the information in Table 5 we note that:

$-0627+532, \quad 0652+577, \quad 1107+485, \quad 1256+546$ and $1311+552$ are $6 \mathrm{C}$ sources (Hales et al. 1993 and references therein).

- $1107+485$ is a member of the DRAO $408 \mathrm{MHz}$ survey (Green \& Riley 1995).
- $1745+670$ and $1753+648$ are members of the NEP survey (Kollgaard et al. 1994).

- $1607+563,1755+578$ and $1815+614$ are members of the $7 \mathrm{C}$ survey (Visser et al. 1995).

- 0514+474 was observed by Leahy \& Roger (1996).

- 0102+480 and 2253+417 are members of the CJ1 survey (Polatidis et al. 1995).

- $1245+676$ is a giant radio galaxy with a GPS core (O’Dea priv. comm. 1996).

- 0140+490 has a large scale symmetric structure 2.5 across.

- 1839+548 and 2119+709 are marked as "quasi-point" sources in JVAS. All other sources are pointlike i.e. they are unresolved by the VLA in "A" configuration at $8.4 \mathrm{GHz}$.

- $1815+614$ and $1946+708$ are CSOs (Taylor et al. 1996; Taylor \& Vermeulen 1997).

\section{Summary}

Gigahertz Peaked Spectrum objects are an astrophysically significant and important class yet they are still not well understood (O'Dea 1998). They are not necessarily a uniform class, and one can easily name subclasses among the whole GPS ensemble. For example CSOs make one well defined group - all of them are GPS galaxies with characteristic VLBI morphologies. It is claimed by Readhead 
Table 5. Some other parameters of JVAS GPS sources

\begin{tabular}{|c|c|c|c|c|c|c|c|c|c|}
\hline Number & B1950name & $\mathrm{S} 4 / \mathrm{S} 5$ & FIRST & B3 & WENSS & CJ2 & Opt. ID & $z$ & Redshift reference \\
\hline 1 & $0001+478$ & & & & & & & & \\
\hline 2 & $0015+529$ & & & & & & & & \\
\hline 3 & $0046+511$ & & & & & & & & \\
\hline 4 & $0051+679$ & & & & & & & & \\
\hline 5 & $0058+498$ & & & & & & & & \\
\hline 6 & $0102+480$ & $\bullet$ & & & & & & & \\
\hline 7 & $0102+511$ & & & & & & & & \\
\hline 8 & $0123+731$ & $\bullet$ & & & & & & & \\
\hline 9 & $0129+560$ & & & & & & & & \\
\hline 10 & $0140+490$ & & & & & & & & \\
\hline 11 & $0148+546$ & & & & & & & & \\
\hline 12 & $0153+389$ & & & & & & & & \\
\hline 13 & $0307+380$ & & & $\bullet$ & & • & QSO & 0.816 & Vermeulen \& Taylor, 1995 \\
\hline 14 & $0335+599$ & & & & & & & & \\
\hline 15 & $0336+473$ & & & & & & & & \\
\hline 16 & $0412+447$ & & & & & & & & \\
\hline 17 & $0454+550$ & & & & & & & & \\
\hline 18 & $0513+714$ & & & & & & & & \\
\hline 19 & $0514+474$ & & & $\bullet$ & & & & & \\
\hline 20 & $0610+510$ & & & & & & QSO & 1.59 & Hook et al., 1996 \\
\hline 21 & $0627+532$ & $\bullet$ & & & & $\bullet$ & QSO & 2.204 & Henstock et al., 1997 \\
\hline 22 & $0630+497$ & • & & & & & & & \\
\hline 23 & $0651+410$ & & & & & • & G & 0.02156 & Marzke et al., 1996 \\
\hline 24 & $0652+577$ & & & & & & & & \\
\hline 25 & $0655+696$ & & & & & & QSO & 1.971 & Moran et al., 1996 \\
\hline 26 & $0718+374$ & & $\bullet$ & & & & & & \\
\hline 27 & $0750+535$ & & • & & & & & & \\
\hline 28 & $0753+373$ & & $\bullet$ & & & & & & \\
\hline 29 & $0753+519$ & & $\bullet$ & & & & QSO & 1.33 & Hook et al., 1996 \\
\hline 30 & $0758+594$ & & & & & & & & \\
\hline 31 & $0849+675$ & & & & & & & & \\
\hline 32 & $0925+745$ & $\bullet$ & & & & & & & \\
\hline 33 & $1017+436$ & & $\bullet$ & & & & QSO & 1.96 & Hook et al., 1996 \\
\hline 34 & $1019+429$ & $\bullet$ & $\bullet$ & $\bullet$ & & & & & \\
\hline 35 & $1032+509$ & & $\bullet$ & & & & & & \\
\hline 36 & $1055+433$ & $\bullet$ & $\bullet$ & $\bullet$ & & & & & \\
\hline 37 & $1107+485$ & $\bullet$ & $\bullet$ & & & & QSO & 0.74 & Hook et al., 1996 \\
\hline 38 & $1125+366$ & & $\bullet$ & & & & & & \\
\hline 39 & $1138+644$ & & & & & & & & \\
\hline 40 & $1206+415$ & & $\bullet$ & & & $\bullet$ & & & \\
\hline 41 & $1226+638$ & & & & & & & & \\
\hline 42 & $1232+366$ & $\bullet$ & $\bullet$ & & & & QSO & 1.60 & Hook et al., 1996 \\
\hline 43 & $1239+606$ & & & & & & & & \\
\hline 44 & $1245+676$ & & & & & & G & 0.103 & Marzke et al., 1996 \\
\hline 45 & $1256+546$ & & $\bullet$ & & & & & & \\
\hline 46 & $1311+552$ & $\bullet$ & $\bullet$ & & & $\bullet$ & QSO & 0.613 & Vermeulen et al., 1996 \\
\hline 47 & $1321+410$ & • & $\bullet$ & & & $\bullet$ & QSO & 0.496 & Vermeulen et al., 1996 \\
\hline 48 & $1338+381$ & $\bullet$ & $\bullet$ & & & & QSO & 3.103 & Hook et al., 1995 \\
\hline 49 & $1357+404$ & & $\bullet$ & & & & & & \\
\hline 50 & $1403+411$ & & • & $\bullet$ & & & & & \\
\hline
\end{tabular}


Table 5. continued

\begin{tabular}{|c|c|c|c|c|c|c|c|c|c|}
\hline Number & B1950name & $\mathrm{S} 4 / \mathrm{S} 5$ & FIRST & B3 & WENSS & CJ2 & Opt. ID & $z$ & Redshift reference \\
\hline 51 & $1454+447$ & & $\bullet$ & & & & & & \\
\hline 52 & $1532+680$ & & & & $\bullet$ & & & & \\
\hline 53 & $1534+501$ & $\bullet$ & $\bullet$ & & & $\bullet$ & QSO & 1.119 & Vermeulen \& Taylor, 1995 \\
\hline 54 & $1544+398$ & $\bullet$ & $\bullet$ & $\bullet$ & & & & & \\
\hline 55 & $1607+563$ & & $\bullet$ & & & & & & \\
\hline 56 & $1627+476$ & & • & & & & & & \\
\hline 57 & $1630+358$ & & $\bullet$ & & & & & & \\
\hline 58 & $1745+670$ & & & & $\bullet$ & & & & \\
\hline 59 & $1753+648$ & & & & $\bullet$ & & & & \\
\hline 60 & $1755+578$ & - & & & $\bullet$ & • & QSO & 2.110 & Henstock et al., 1997 \\
\hline 61 & $1801+459$ & & & $\bullet$ & & & & & \\
\hline 62 & $1815+614$ & & & & $\bullet$ & $\bullet$ & QSO & 0.601 & Vermeulen \& Taylor, 1995 \\
\hline 63 & $1820+397$ & $\bullet$ & & $\bullet$ & & & & & \\
\hline 64 & $1839+548$ & & & & & & & & \\
\hline 65 & $1946+708$ & $\bullet$ & & & $\bullet$ & • & G & 0.101 & Stickel \& Kühr, 1993 \\
\hline 66 & $2000+472$ & & & & & & & & \\
\hline 67 & $2005+642$ & & & & $\bullet$ & $\bullet$ & QSO & 1.574 & Henstock et al., 1997 \\
\hline 68 & $2013+508$ & & & & & & & & \\
\hline 69 & $2014+463$ & & & & & & & & \\
\hline 70 & $2119+709$ & & & & & & & & \\
\hline 71 & $2151+431$ & & & & & & & & \\
\hline 72 & $2248+555$ & & & & & & & & \\
\hline 73 & $2253+417$ & - & & & & & QSO & 1.476 & Hewitt \& Burbidge, 1989 \\
\hline 74 & $2310+385$ & & & & & $\bullet$ & QSO & 2.17 & Hewitt \& Burbidge, 1989 \\
\hline 75 & $2341+697$ & & & & & & & & \\
\hline 76 & $2356+385$ & $\bullet$ & & & & $\bullet$ & QSO & 2.704 & Stickel \& Kühr, 1994 \\
\hline
\end{tabular}

References of the catalogues:

S4 - Pauliny-Toth et al., 1978.

S5 - Kühr et al., 1981b.

FIRST - White et al., 1997.

B3 - Ficarra et al., 1985.

WENSS - Rengelink et al., 1997.

CJ2 - Taylor et al., 1994.

et al. (1996b) that CSOs play a key role as an initial stage in the evolutionary scenario of radio-loud AGNs.

Another fascinating subset of GPS class are objects with extreme $(z>3)$ redshifts. There are 9 such objects in the "working sample" (O'Dea, priv. comm. 1996). Additionally there are $\sim 20$ objects with high $(1<z<3)$ redshifts. All the objects with $z>1$ are identified with quasars.

The above two issues alone are already a good argument to extend the number of known GPS radio sources. With such a goal in mind we have made a search for candidate GPS sources in the part of the Jodrell Bank-VLA Astrometric Survey limited to $35^{\circ} \leq \delta \leq 75^{\circ}$, namely we compared $8.4 \mathrm{GHz}$ flux densities derived from JVAS with respective $1.4 \mathrm{GHz}$ and $5 \mathrm{GHz}$ fluxes in available catalogues. We treated a source as a plausible candidate if its spectrum seemed to be convex according to the criterion we had arbitrarily assumed.
Quite expectedly some of the candidates selected in this manner are already recognised as GPS objects so we did not deal with them here. Using flux densities at low frequencies i.e. $365 \mathrm{MHz}$ and sometimes even $151 \mathrm{MHz}$ in available catalogues we were able to classify 24 objects as GPS sources without any further measurements. For the majority of selected objects the flux densities at frequencies well below $1 \mathrm{GHz}$ were not available. In these cases we performed observations with MERLIN at $408 \mathrm{MHz}$ to establish the low-frequency part of the spectra.

Our final decision on which of our candidates are and which are not GPS sources has been made based on our MERLIN data (or Texas catalogue when available), NVSS, GB6 and JVAS catalogues. Combining flux density measurements made with high resolution both at low (MERLIN, $408 \mathrm{MHz}$ ) and high (VLA, 8.4 GHz) frequencies enabled us to eliminate the effects of confusion and any contribution from possible extended "halos". 
Table 6. Candidate GPS sources

\begin{tabular}{ll}
\hline B1950name & Reason \\
\hline $0001+478$ & GB flux is 73\% greater than NVSS flux \\
$0046+511$ & no 2nd epoch data \\
$0051+679$ & GB flux is 36\% greater than NVSS flux \\
$0058+498$ & no 2nd epoch data \\
$0307+380$ & poor fit to the broken power-law curve \\
$0412+447$ & GB flux is 4.7 times greater than NVSS flux, inverted spectrum only \\
$0651+410$ & no 2nd epoch data \\
$0655+696$ & GB flux is 55\% less than NVSS flux \\
$0718+374$ & no 2nd epoch data \\
$0753+519$ & no 2nd epoch data \\
$0758+594$ & GB flux is 34\% less than NVSS flux \\
$1017+436$ & no 2nd epoch data \\
$1055+433$ & B3 flux (420 mJy) and MERLIN 408 MHz flux (182 mJy) do not match \\
$1125+366$ & FIRST flux does not match NVSS and GB fluxes, inverted spectrum only \\
$1206+415$ & FIRST flux does not match NVSS and GB fluxes \\
$1232+366$ & FIRST flux matches NVSS flux but they both don't match GB and S4 fluxes - \\
& extended component? \\
$1357+404$ & FIRST flux does not match NVSS and GB fluxes, inverted spectrum only \\
$1544+398$ & B3 flux matches MERLIN flux but FIRST flux does not match NVSS and GB fluxes \\
$1839+548$ & no 2nd epoch data \\
$2005+642$ & inverted spectrum only \\
$2013+508$ & GB flux is 48\% less than NVSS flux \\
$2014+463$ & no 2nd epoch data \\
$2248+555$ & no 2nd epoch data \\
$2341+697$ & no 2nd epoch data \\
\hline
\end{tabular}

We regarded a source as a GPS if it had fitted well a "broken power-law" function and was not variable.

The sample we present here is the largest single contribution to the pool of known GPS sources collected so far. Only 3 of our sources $(0513+714,0758+594,1946+708)$ are overlapping with another large collection of GPS sources, namely with the WENSS based sample ${ }^{7}$ (SSB98). Seven sources in our sample have large redshifts $(z \gtrsim 2)$; the largest one is $z=3.103$.

Our approach to finding GPS sources was to search from high frequency to lower ones. WENSS has been equally successful in defining GPS sources but searching from low frequencies to higher ones. We want to stress though that our approach can successfully be applied to the areas of the sky not planned to be covered by WENSS $\left(\delta<30^{\circ}\right)$ but already covered by the other catalogues we used (NVSS, GB6, JVAS). For the part of JVAS we used so far (Patnaik et al. 1992) we found that $9 \%$ of JVAS sources are GPS; therefore the whole JVAS encompassing around 3000 sources could easily yield $250-300$ of such objects.

\footnotetext{
${ }^{7} 0513+714$ and $0758+594$ are not marked in Table 5 as WENSS sources because these objects are not mentioned by Rengelink et al. (1997). On the other hand, except 1946+708, the WENSS objects we list in Table 5 have not been recognised as GPS by SSB98.
}

Acknowledgements. The initial stages of the programme described in this paper were completed when AM stayed at MPIfR in Bonn. The Max-Planck-Gesellschaft stipend which supported him in that time is gratefully acknowledged.

AM acknowledges support from the Polish State Committee for Scientific Research grant 2.P304.003.07 and EU grant ERBCIPDCT940087.

HF is supported in part by the DFG, grant Fa 358/1-1\&2.

We thank the NVSS team led by Jim Condon for making NVSS public domain data prior its final publication. Special thanks to Bill Cotton for his co-operation.

This research has made use of the NASA/IPAC Extragalactic Database (NED) which is operated by the Jet Propulsion Laboratory, California Institute of Technology, under contract with the National Aeronautics and Space Administration.

\section{References}

Baars J.W.M., Genzel R., Pauliny-Toth I.I.K., Witzel A., 1977, A\&A 61,99

Cersosimo J.C., Lebron Santos M., Cintrón S.I., Quiniento Z.M., 1994, ApJS 95, 157

Condon J.J., Cotton W.D., Greisen E.W., et al., 1998, AJ 115, 1693

Douglas J.N., Bash F.N., Arakel Bozyan F., Torrence G.W., 1996, AJ 111, 1945

Ficarra A., Grueff G., Tomasetti G., 1985, A\&AS 59, 255

Gopal-Krishna, Patnaik A.R., Steppe H., 1983, A\&A 123, 107 
Gopal-Krishna, Spoelstra T.A.Th., 1993, A\&A 271, 103

Green D.A., Riley J.M., 1995, MNRAS 274, 324

Gregory P.C., Scott W.K., Douglas K., Condon J.J., 1996, ApJS 103, 427

Hales S.E.G., Baldwin J.E., Warner P.J., 1993, MNRAS 263, 25

Henstock D.R., Browne I.W.A., Wilkinson P.N., McMahon R.G., 1997, MNRAS 290, 380

Hewitt A., Burbidge G., 1989, magnetic tape

Hook I.M., McMahon R.G., Patnaik A.R., et al., 1995, MNRAS 273,63

Hook I.M., McMahon R.G., Irwin M.J., Hazard C., 1996, MNRAS 282, 1274

Kollgaard R.I., Brinkmann W., McMath Chester M., et al., 1994, ApJS 93, 145

Kühr H., Witzel A., Pauliny-Toth I.I.K., Nauber U., 1981a, A\&AS 45, 367

Kühr H., Pauliny-Toth I.I.K., Witzel A., Schmidt J., 1981b, AJ 86, 854

Leahy D.A., Roger R.S., 1996, A\&AS 115, 345

Marzke R.O., Huchra J.P., Geller M.J., 1996, AJ 112, 1803

Moffet A.T., 1975, in: Sandage A., Sandage M., Kristian J. (eds.) Stars and Stellar Systems, Vol. IX, p. 211

Moran E.C., Helfand D.J., Becker R.H., White R.L., 1996, ApJ 461,127

O’Dea C.P., Baum S.A., Stanghellini C., 1991, ApJ 380, 66 (OBS91)

O'Dea C.P., 1998, PASP 110, 493

Patnaik A.R., Browne I.W.A., Wilkinson P.N., Wrobel J.M., 1992, MNRAS 254, 655

Pauliny-Toth I.I.K., Witzel A., Preuss E., et al., 1978, AJ 83, 451

Polatidis A.G., Wilkinson P.N., Xu W., et al., 1995, ApJS 98, 1

Rengelink R.B., Tang Y., de Bruyn A.G., et al., 1997, A\&A 124, 259

Readhead A.C.S., Taylor G.B., Xu W., et al., 1996a, ApJ 460, 612
Readhead A.C.S., Taylor G.B., Pearson T.J., Wilkinson P.N., 1996b, ApJ 460, 634

Snellen I.A.G., Zhang M., Schilizzi R.T., et al., 1995, A\&A 300, 359

Snellen I.A.G., Schilizzi R.T., de Bruyn A.G., et al., 1998, A\&AS 131, 435 (SSB98)

Spoelstra T.A.Th., Patnaik A.R., Gopal-Krishna, 1985, A\&A 152,38

Stanghellini C., O'Dea C.P., Baum S.A., Fanti R., 1990, Compact Steep Spectrum \& Gigahertz-Peaked Spectrum Sources, Dwingeloo Workshop, 18-19 June 1990, Fanti C., Fanti R., O'Dea C.P., Schilizzi R.T. (eds.)

Stanghellini C., Dallacasa D., O'Dea C.P., et al., 1996, 2nd workshop on GPS and CSS Radio Sources, Leiden, 30 Sept. -2 Oct. 1996, Snellen I.A.G., Schilizzi R.T., Röttgering H.J.A., Bremer M.N. (eds.)

Stickel M., Kühr H., 1993, A\&AS 100, 395

Stickel M., Kühr H., 1994, A\&AS 105, 67

Taylor G.B., Vermeulen R.C., 1997, ApJ 485, 9

Taylor G.B., Vermeulen R.C., Pearson T.J., et al., 1994, ApJS 95,345

Taylor G.B., Vermeulen R.C., Readhead A.C.S., et al., 1996, 2nd workshop on GPS and CSS Radio Sources, Leiden, 30 Sept. - 2 Oct. 1996, Snellen I.A.G., Schilizzi R.T., Röttgering H.J.A., Bremer M.N. (eds.)

Vermeulen R.C., Taylor G.B., 1995, AJ 109, 1983

Vermeulen R.C., Taylor G.B., Readhead A.C.S., Browne I.W.A., 1996, AJ 111, 1013

Visser A.E., Riley J.M., Röttgering H.J.A., Waldram E.M., 1995, A\&AS 110, 419

de Vries W.H., Barthel P.D., O’Dea C.P., 1997, A\&A 321, 105

Walker R.C., 1981, AJ 86, 1323

Wilkinson P.N., Polatidis A.G., Readhead A.C.S., Xu W., Pearson T.J., 1994, ApJ 432, L87

White R.L., Becker R.H., 1992, ApJS 79, 331 (WB92)

White R.L., Helfand D.J., Becker R.H., Gregg M.D., 1997, ApJ 475,479 
1.) $0059+581$

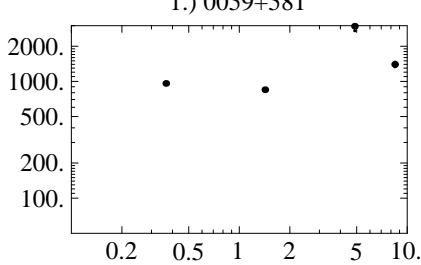

4.) $0338+480$

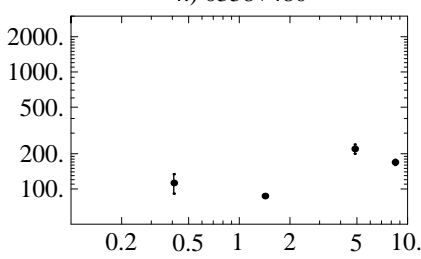

7.) $0621+446$

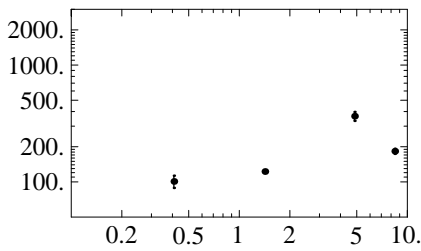

10.) $0708+742$

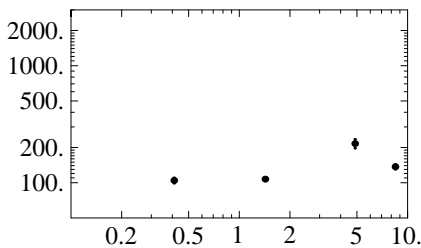

13.) $0851+719$

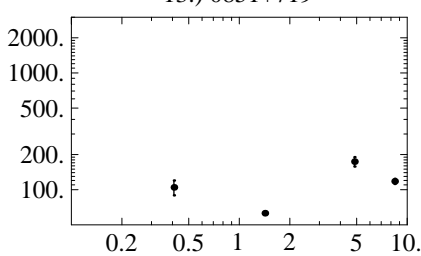

16.) $1035+430$

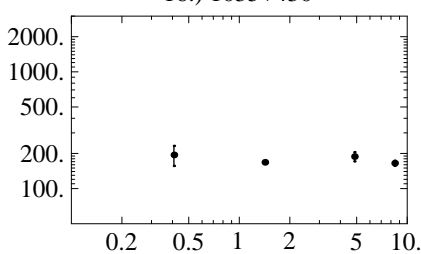

19.) $1239+552$

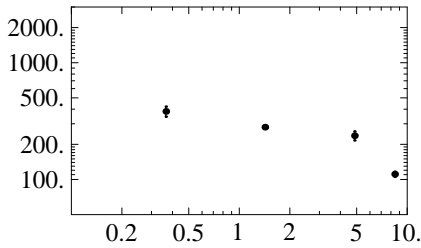

22.) $1320+394$

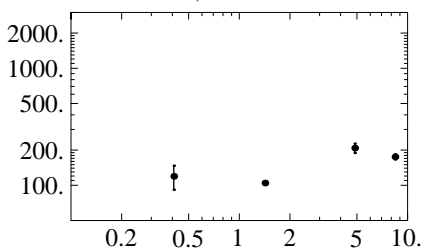

2.) $0129+431$

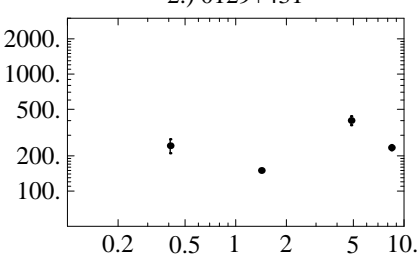

5.) $0533+446$

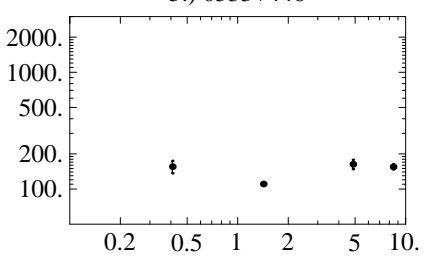

8.) $0652+426$

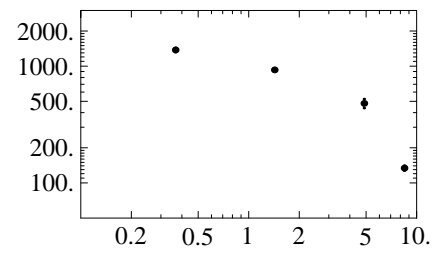

11.) $0713+669$

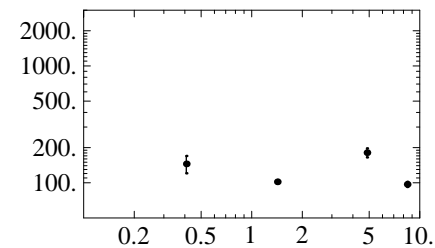

14.) $0924+732$

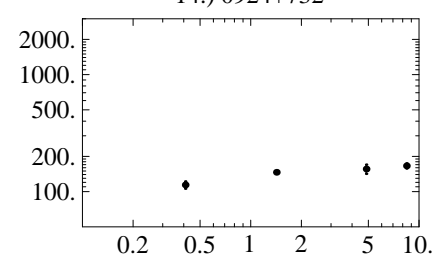

17.) $1043+541$

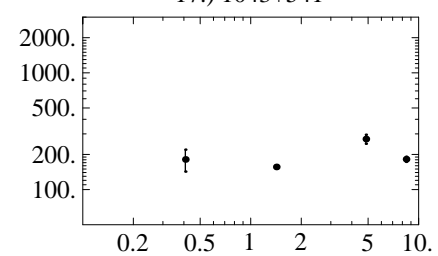

20.) $1300+485$

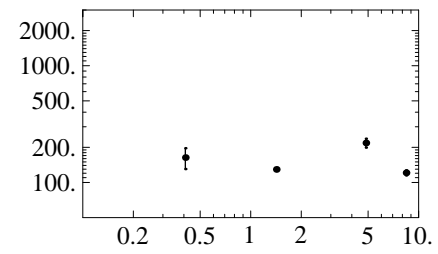

23.) $1324+574$

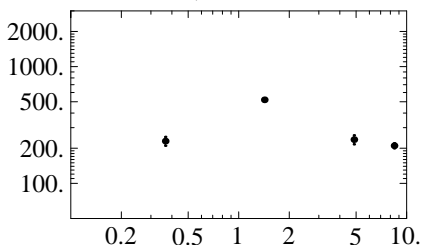

3.) $0213+444$

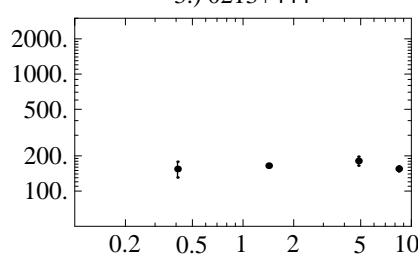

6.) $0559+422$

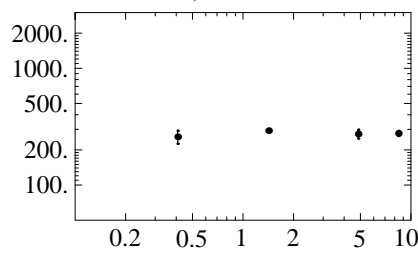

9.) $0700+470$

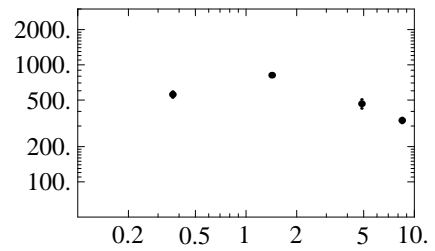

12.) $0828+493$

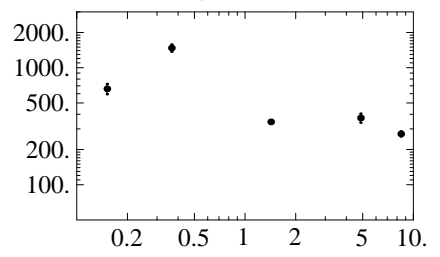

15.) $0939+620$

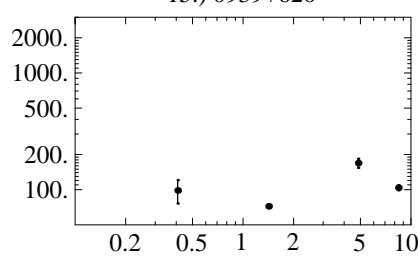

18.) $1101+609$

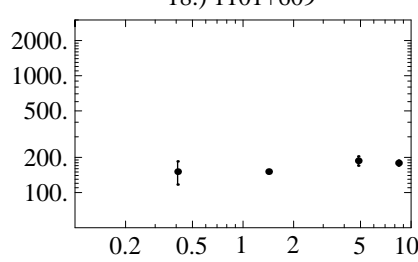

21.) $1308+471$
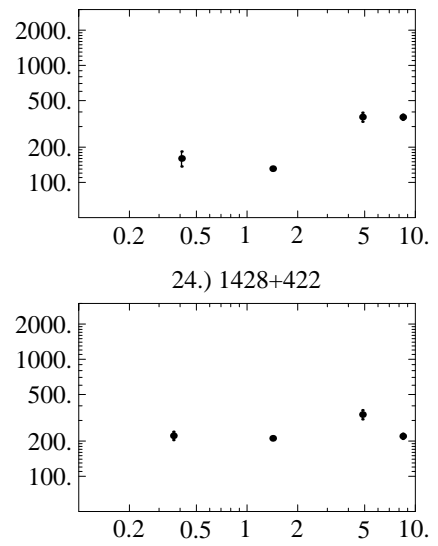

Fig. 1. Spectra of non-GPS sources. Abscissae: frequency in $\mathrm{GHz}$, ordinates: flux densities in mJy 

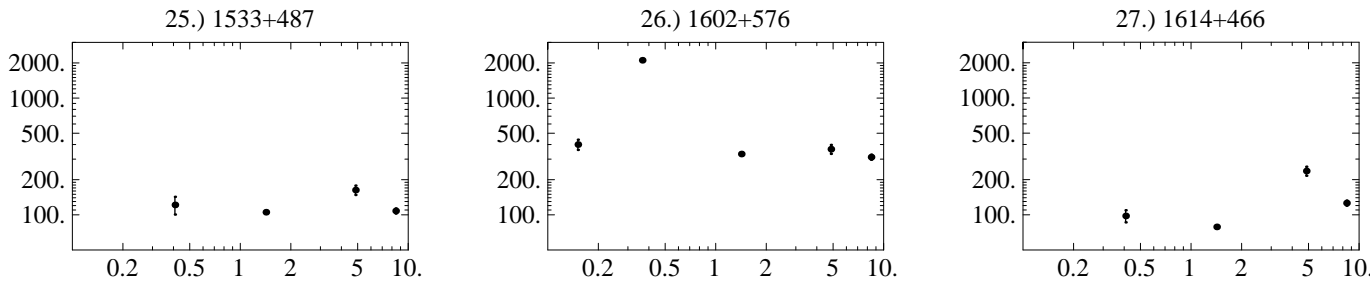

37.) $2323+478$

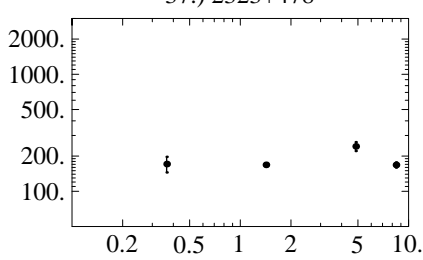

Fig. 1. continued

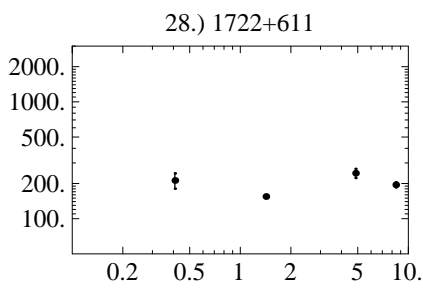

29.) $1724+609$

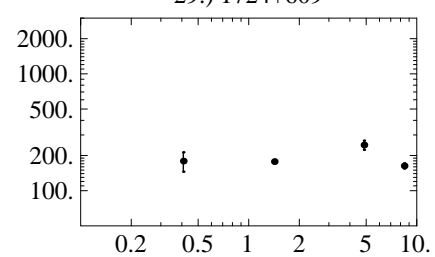

32.) $1839+389$

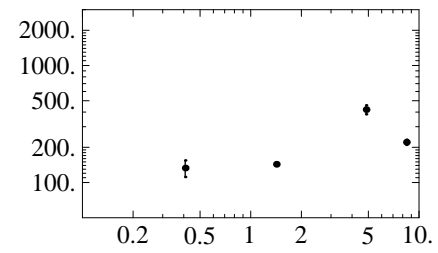

34.) $1941+413$
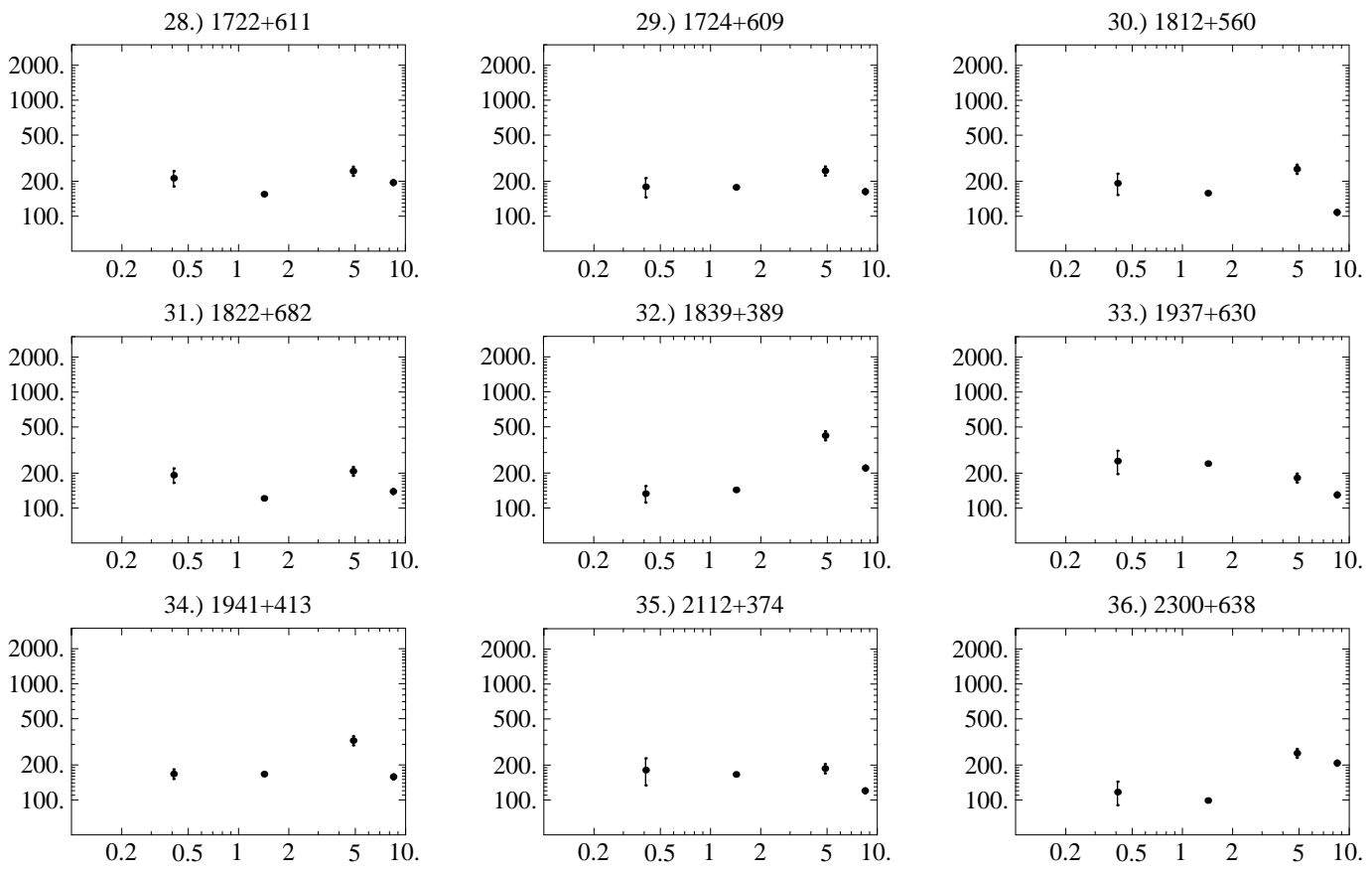

33.) $1937+630$
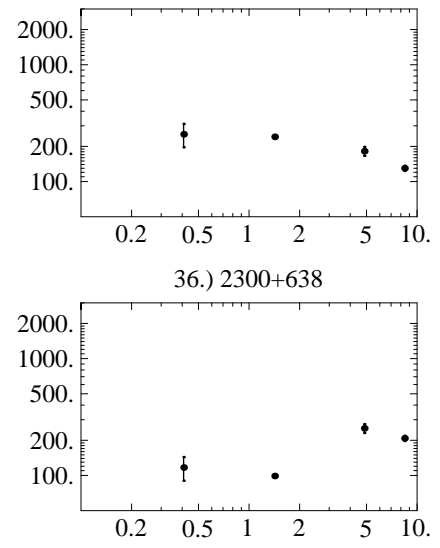


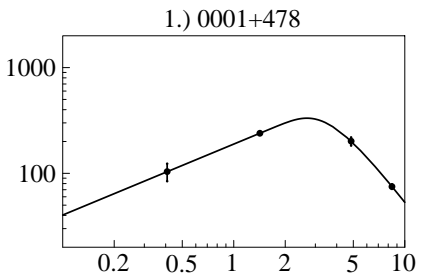

4.) $0051+679$

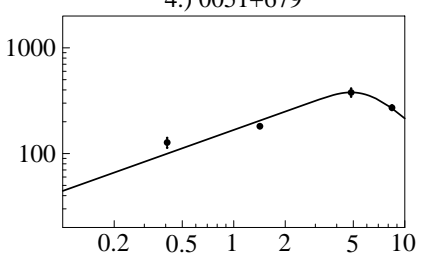

7.) $0102+511$

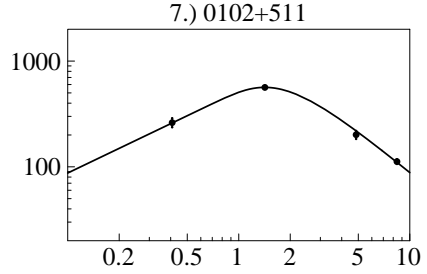

10.) $0140+490$

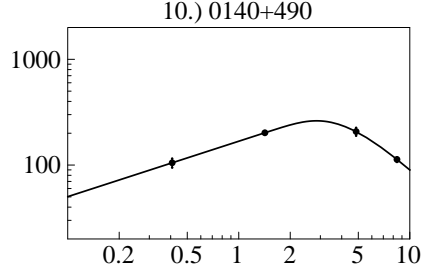

13.) $0307+380$

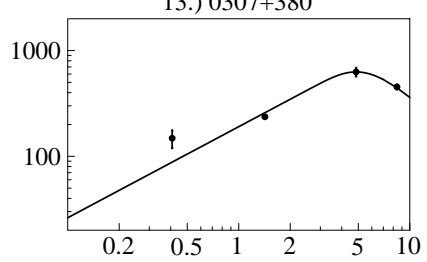

16.) $0412+447$

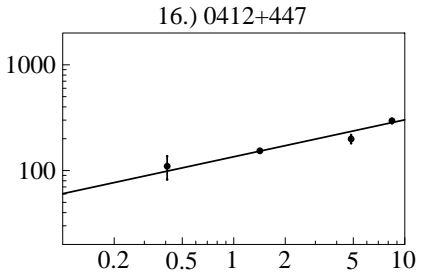

19.) $0514+474$

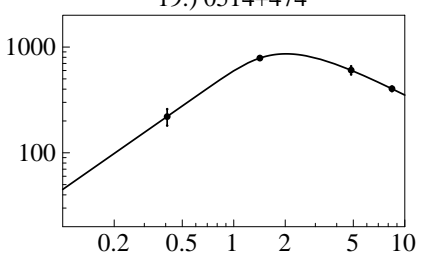

22.) $0630+497$

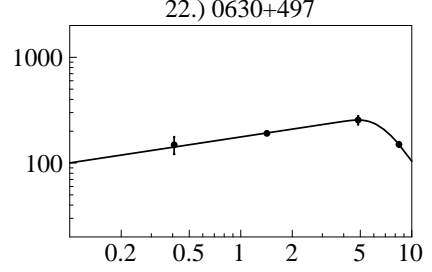

2.) $0015+529$

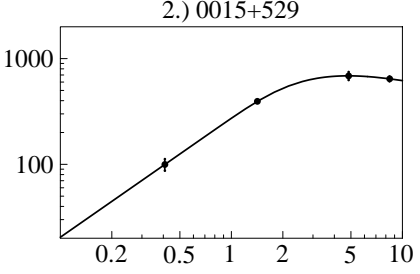

5.) $0058+498$

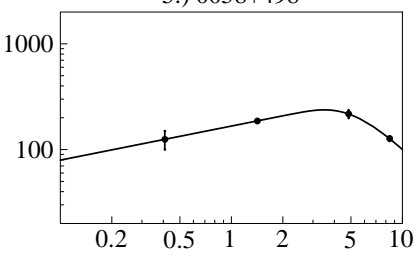

8.) $0123+731$

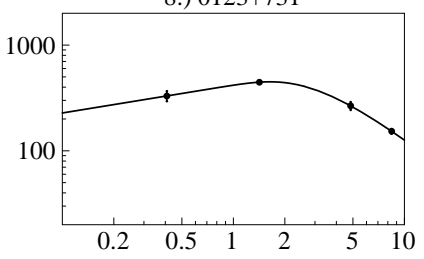

11.) $0148+546$

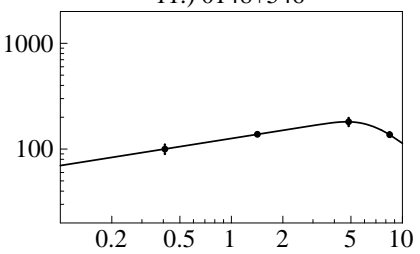

14.) $0335+599$

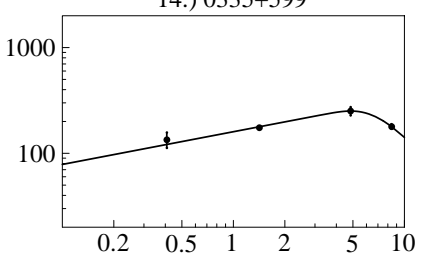

17.) $0454+550$

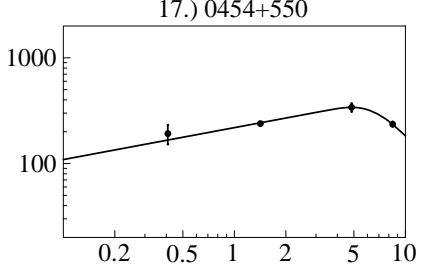

20.) $0610+510$

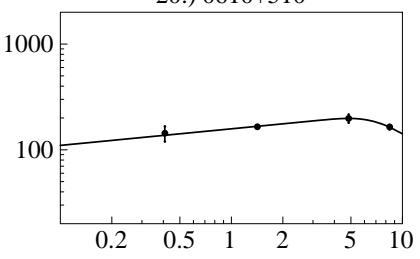

23.) $0651+410$

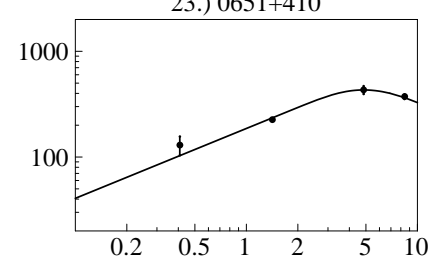

3.) $0046+511$

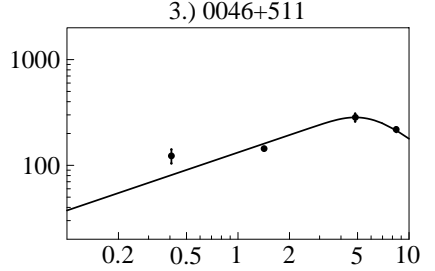

6.) $0102+480$

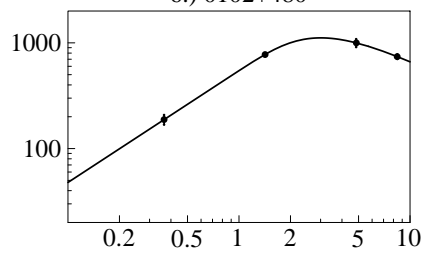

9.) $0129+560$

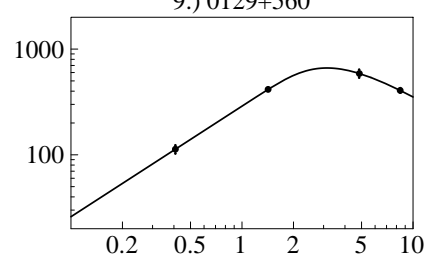

12.) $0153+389$

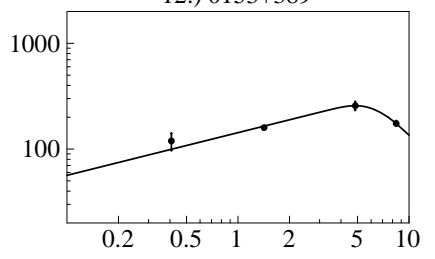

15.) $0336+473$

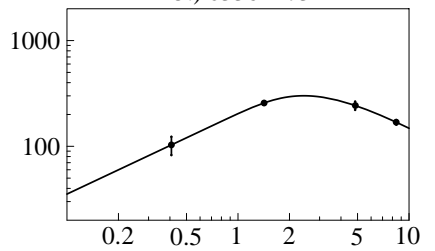

18.) $0513+714$

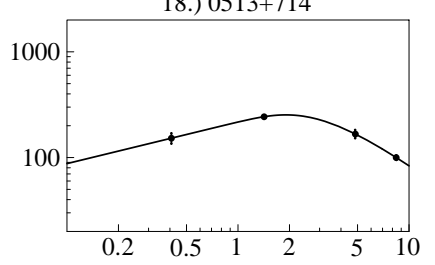

21.) $0627+532$

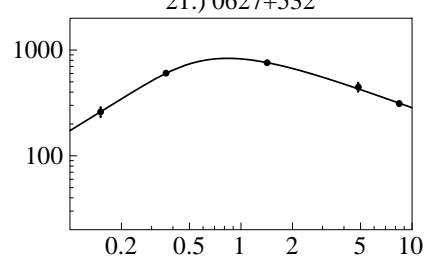

24.) $0652+577$

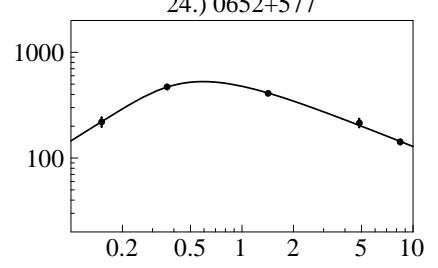

Fig. 2. Spectra of GPS sources. Abscissae: frequency in GHz, ordinates: flux densities in mJy 


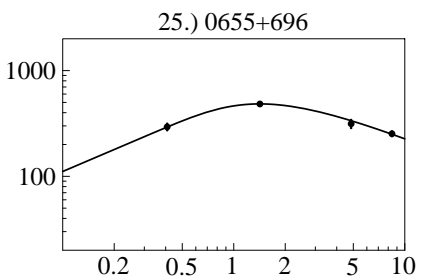

28.) $0753+373$

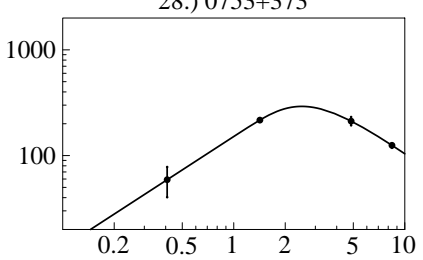

31.) $0849+675$

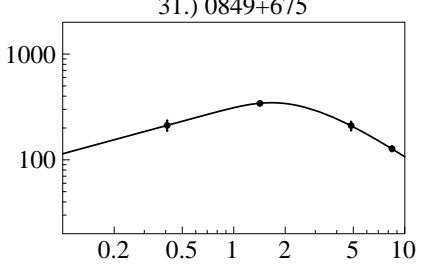

34.) $1019+429$

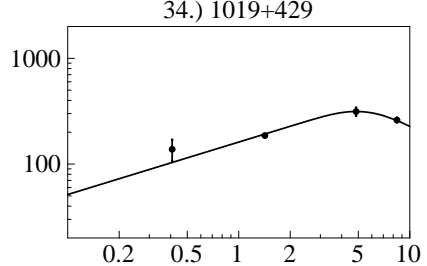

37.) $1107+485$

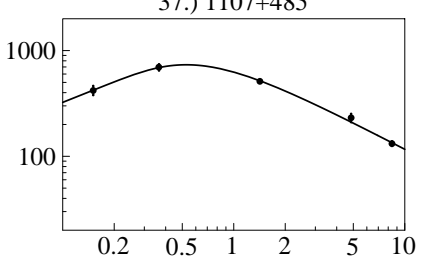

40.) $1206+415$

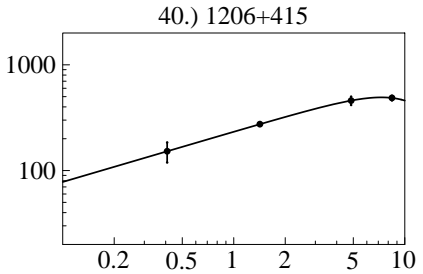

43.) $1239+606$
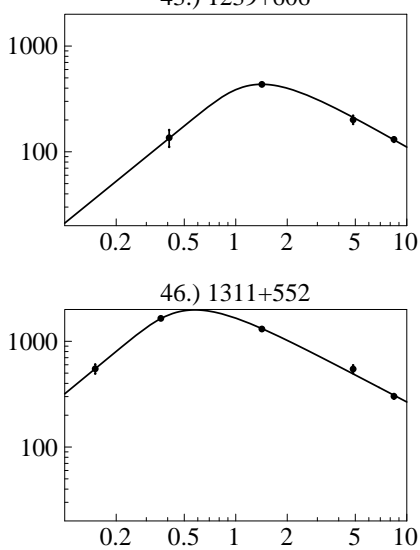

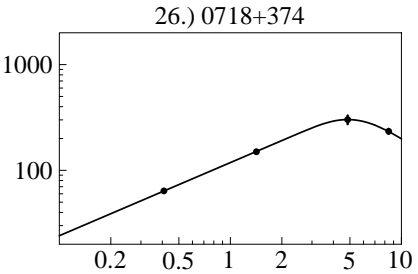

29.) $0753+519$

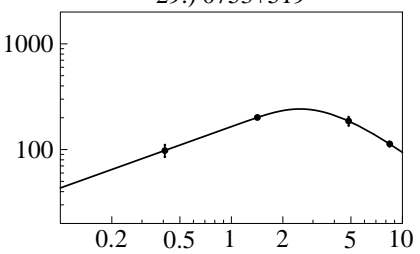

32.) $0925+745$

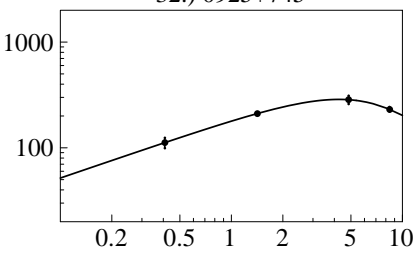

35.) $1032+509$

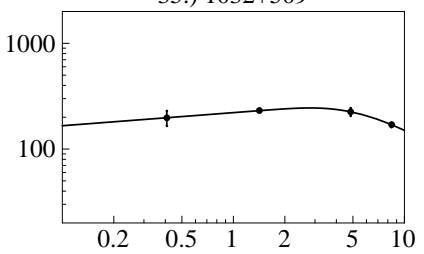

38.) $1125+366$

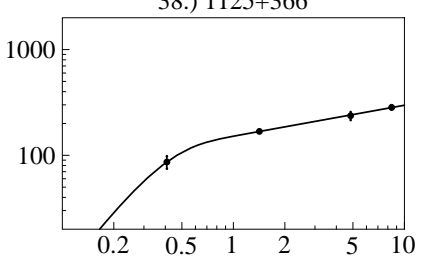

41.) $1226+638$

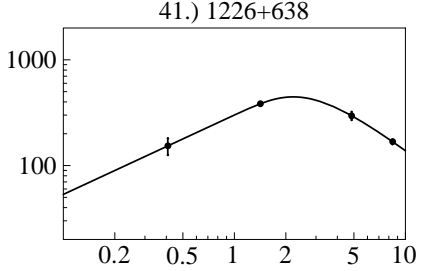

44.) $1245+676$

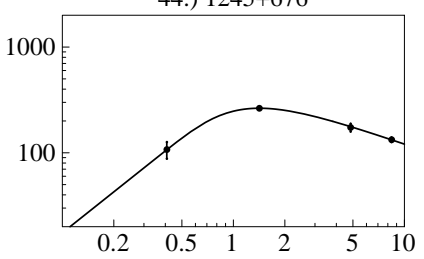

47.) $1321+410$

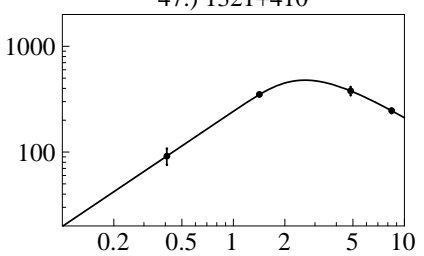

27.) $0750+535$

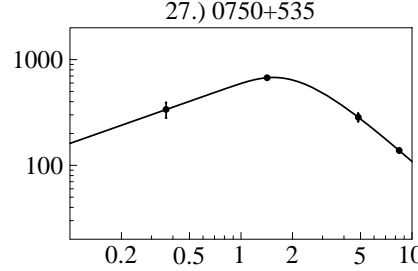

30.) $0758+594$

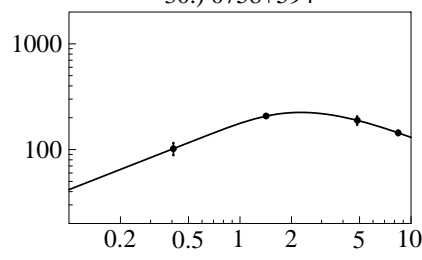

33.) $1017+436$

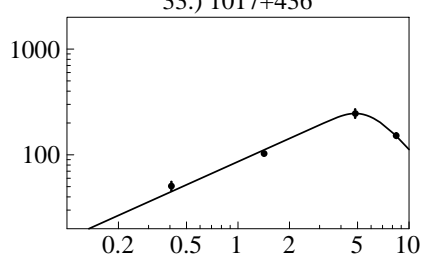

36.) $1055+433$

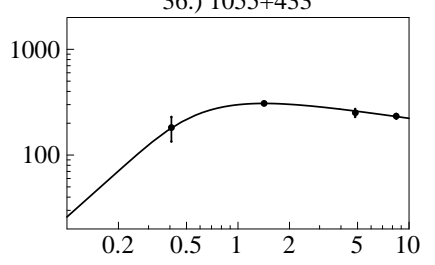

39.) $1138+644$

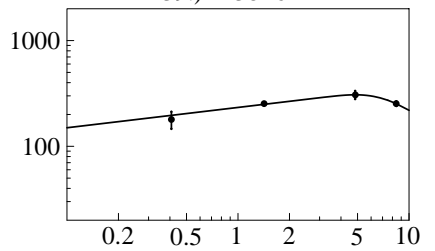

42.) $1232+366$

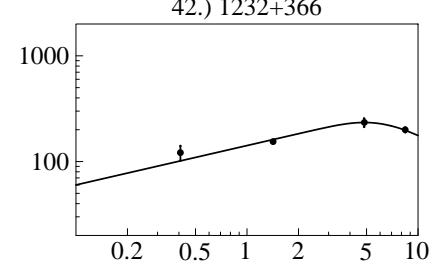

45.) $1256+546$

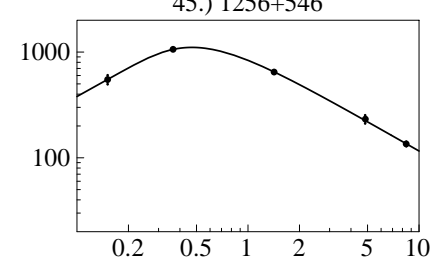

48.) $1338+381$

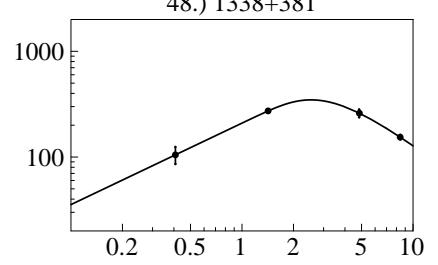

Fig. 2. continued 


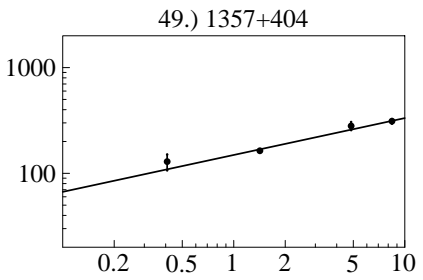

52.) $1532+680$

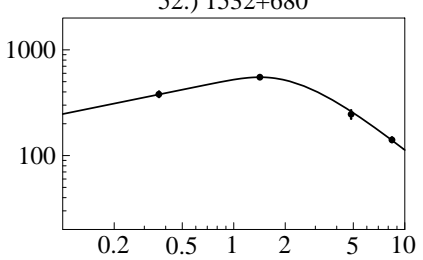

55.) $1607+563$

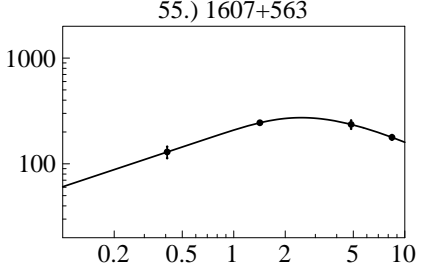

58.) $1745+670$

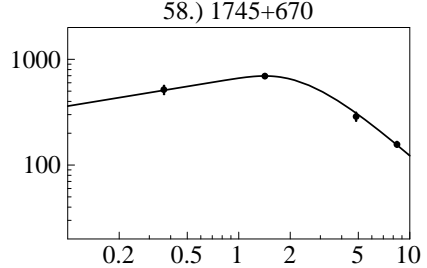

61.) $1801+459$

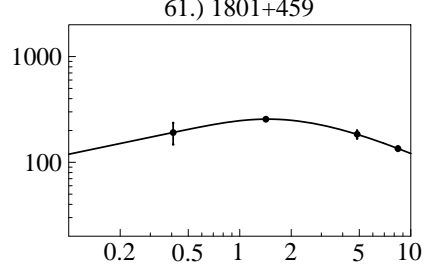

64.) $1839+548$

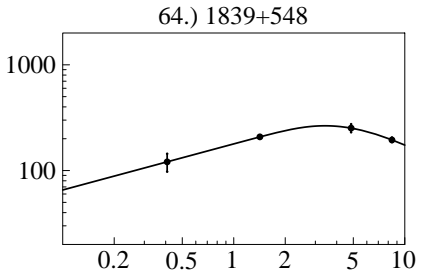

67.) $2005+642$

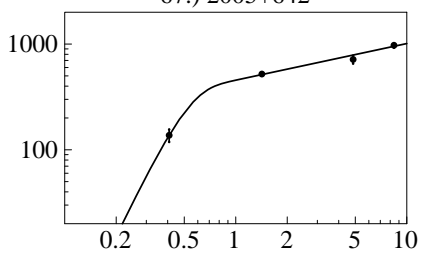

70.) $2119+709$

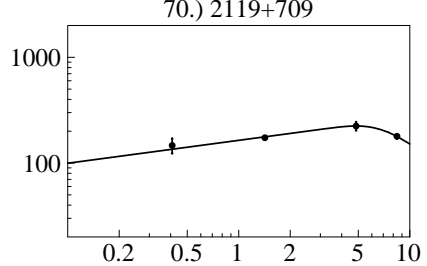

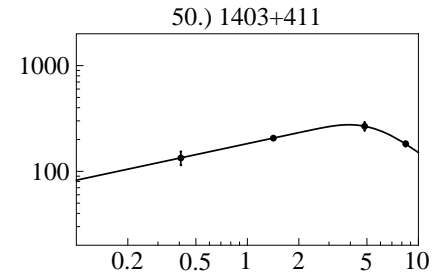

53.) $1534+501$

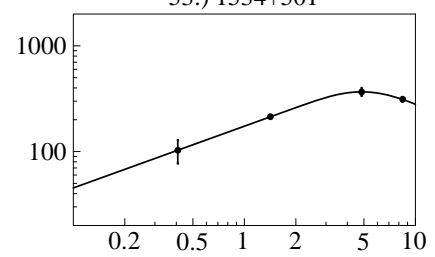

56.) $1627+476$

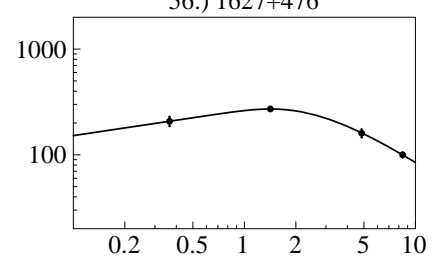

59.) $1753+648$

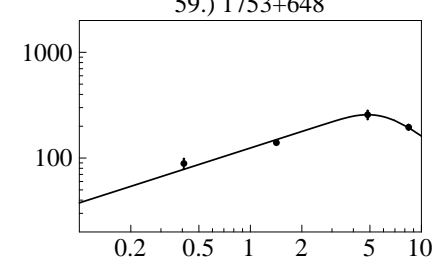

62.) $1815+614$

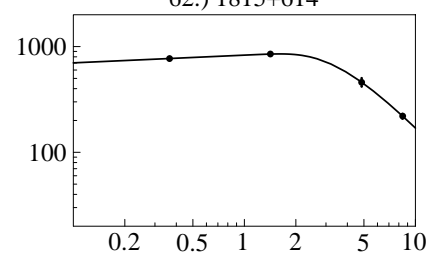

65.) $1946+708$

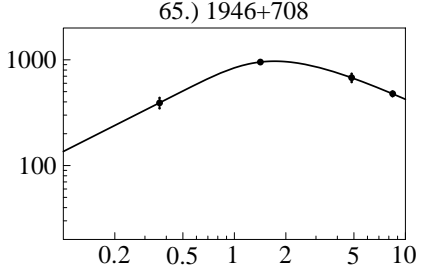

68.) $2013+508$

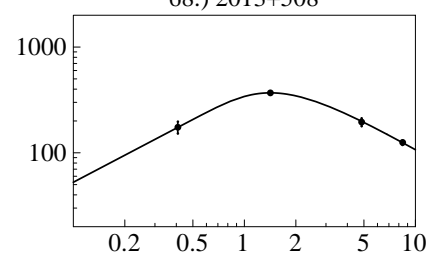

71.) $2151+431$

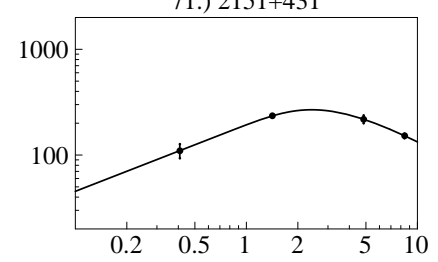

51.) $1454+447$

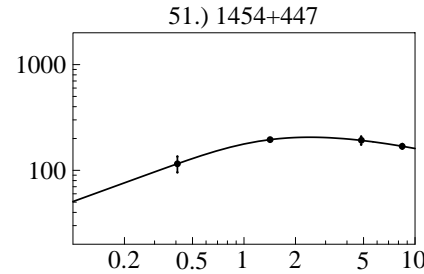

54.) $1544+398$

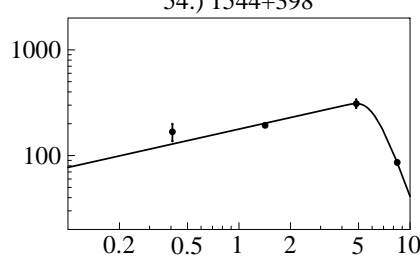

57.) $1630+358$

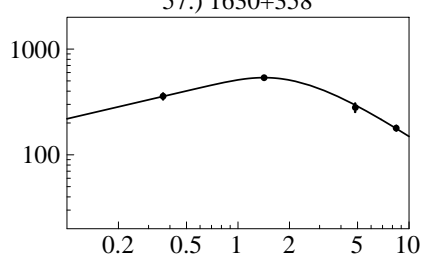

60.) $1755+578$

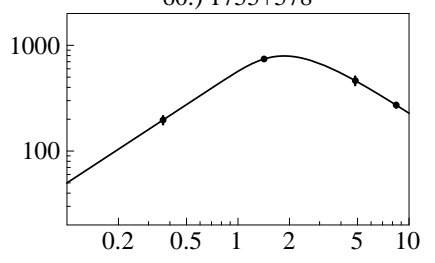

63.) $1820+397$

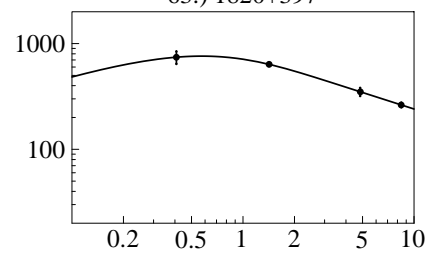

66.) $2000+472$

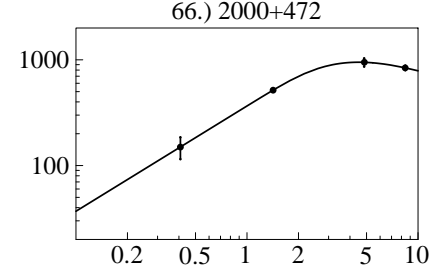

69.) $2014+463$

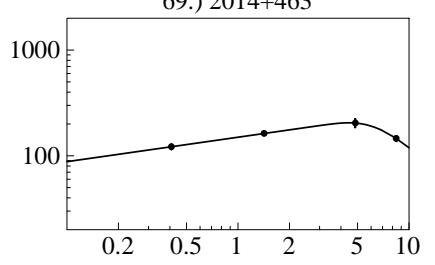

72.) $2248+555$

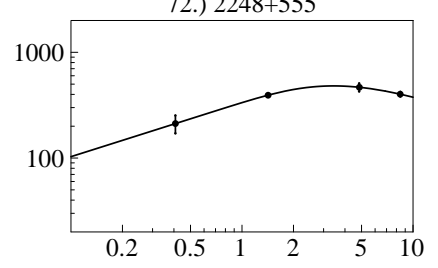

Fig. 2. continued 
A. Marecki et al.: Gigahertz Peaked Spectrum sources from the Jodrell Bank-VLA Astrometric Survey
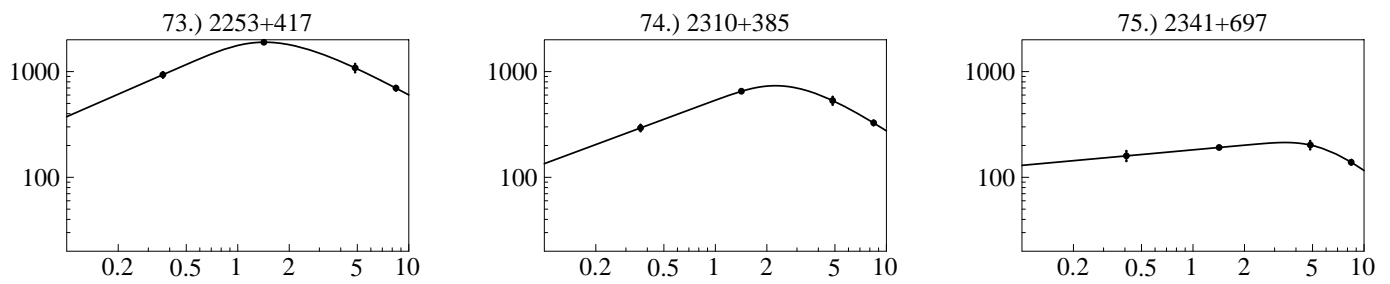

76.) $2356+385$

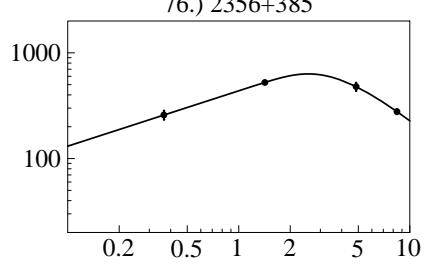

Fig. 2. continued 